\title{
Stochastic traveling wave solution to stochastic generalized KPP equation
}

\author{
Zhehao Huang and Zhengrong Liu
}

\begin{abstract}
In this paper, we consider a stochastic generalized KPP equation driven by a white noise term. Denote $u$ the solution to the equation with Heaviside initial condition $u_{0}(x)=\chi_{(-\infty, 0]}(x)$. Choosing a suitable marker of wavefront $R(t)$, we prove that $u(t, \cdot+R(t))$ is a stationary process and $\lim _{t \rightarrow \infty} R(t) / t$ exists almost surely, which verify the existence of stochastic traveling wave solution to the equation.
\end{abstract}

Mathematics Subject Classification (2010). Primary 99Z99;

Secondary 00A00.

Keywords. Wavefront, Stationary process, Stochastic traveling wave solution, Stochastic generalized KPP equation.

\section{Introduction}

The Kolmogorov-Petrovsky-Piscunov (KPP) equation $[13,20]$

$$
\frac{\partial}{\partial t} u=\frac{D}{2} u_{x x}+R u(K-u), \quad x \in \mathbf{R}, t \geq 0,
$$

where $R>0$ is the reproduction rate, $K>0$ the carrying capacity, and $D>0$ the diffusion coefficient, provides a deterministic model for the density $u$ of a population living in an environment with a limited carrying capacity. The normalization of (1.1),

$$
\frac{\partial}{\partial t} u=u_{x x}+u(1-u)
$$

is perhaps the simplest equation which possesses the traveling wave solutions. In fact, (1.2) has a solution $u(t, x)=\phi_{c}(x-c t)$ for any $c \geq 2$, where the function $\phi_{c}(\xi)$ converges exponentially to 1 as $\xi \rightarrow-\infty$ and to 0 as $\xi \rightarrow \infty$. In the classical paper [20], Kolmogorov et al. proved that if $u$ is the solution

Supported by the National Natural Science Foundation (No.11171115). 
to $(1.2)$ with Heaviside initial condition, $u(0, x)=\chi_{(-\infty, 0]}(x)$, then there is a function $m(t), t>0$ with the property

$$
\lim _{t \rightarrow \infty} \frac{m(t)}{t}=2
$$

and

$$
\lim _{t \rightarrow \infty} \sup _{\xi \in \mathbf{R}}\left|u(\xi+m(t), t)-\phi_{2}(\xi)\right|=0 .
$$

Many authors $[2,4,24,37,45]$ have paid attention to (1.1) and extended the results of $[13,20]$ to more general equations, especially the delayed versions $[3,15,36,46]$.

A system in reality is usually of uncertainty due to some external noise, which is random. The random effects are considered not only as compensations for the detects in some deterministic models, but also rather essential phenomena $[1,5,6,10,18,26-28,47]$. Therefore, one should make the model (1.1) more realistic by considering environmental noise. As $[10,12,34,35]$, the carrying capacity is supposed to be stochastic and is given as the form depending on time, $K(t)=c_{0}+k(t) \zeta_{t}$, where $c_{0}>0$ and $\zeta_{t}$ is the white noise. Substituting $K$ into (1.1) gives a stochastic partial differential equation

$$
d u(t, x)=\left(\frac{D}{2} u_{x x}(t, x)+R u(t, x)\left(c_{0}-u(t, x)\right)\right) d t+k(t) u(t, x) d W_{t},
$$

where $W_{t}$ is the Brownian motion whose distributional time derivative is $\zeta_{t}$. There are other ways introducing environmental noise in (1.5). In the spatially homogeneous case, $D=0$, different versions were discussed and compared in $[14,21,23,32,33,41]$. The case when the carrying capacity $K>0$ is constant and $R=r_{0}+k \zeta_{t}$ has been analysed in [25]. Some works discussed whether the Itô or Stratonovich interpretation of the equation is most appropriate.

In [39], the authors considered the KPP equation driven by a particular space-time white noise term

$$
\left\{\begin{array}{l}
\frac{\partial}{\partial t} u=u_{x x}+\theta u-u^{2}+\sqrt{u} \zeta_{t, x} \\
u(0, x)=1 \wedge(-x \vee 0)
\end{array}\right.
$$

The form of the noise term in (1.6) arises from particle branching in a particle approximation. The same term which appears in the stochastic partial differential equation describes the density of one dimensional super Brownian motion [22]. It has been shown that (1.6) arises as the high density limit of particle systems which undergo branching random walks and have an extra death mechanism due to overcrowding. The authors proved that there exist stochastic wavelike solutions which travel with a linear speed. In fact, they looked for solutions $u$ to (1.6) which satisfy that the wavefront $R_{0}(u(t)):=\sup \{x \in \mathbf{R}: u(t, x)>0\} \in(-\infty, \infty)$ for all $t \geq 0, u\left(t, \cdot+R_{0}(u(t))\right)$ is a stationary process in time and $\lim _{t \rightarrow \infty} R_{0}(u(t)) / t$ exists almost surely. The work related to the compact support property of solutions to the equation [31]. Such a solution is called a stochastic traveling wave solution. 
In $[7,29,30]$, the authors considered another version of stochastic KPP equation

$$
\left\{\begin{array}{l}
\frac{\partial}{\partial t} u=u_{x x}+\theta u-u^{2}+\varepsilon \sqrt{u(1-u)} \zeta_{t, x} \\
0 \leq u(0, x) \leq 1 \\
u(0, x)=1, x<-n \\
u(0, x)=0, x>n,
\end{array}\right.
$$

where $n>0$ is a constant, $\zeta_{t, x}$ is also a two-parameter white noise. Note that (1.7) has two different trivial equilibrium solutions, $u=0$ and $u=1$, which is different from (1.6). In [29], the authors showed that for $\varepsilon$ small enough, $\lim _{t \rightarrow \infty} R_{0}(u(t)) / t$ exists and lies in $(0, \infty)$. The limit depends only on $x$. The law of $u\left(t, \cdot+R_{0}(u(t))\right)$ tends to a stationary limit as $t \rightarrow \infty$. The authors also analyzed the region which is the smallest closed interval containing the points $x$ at which $0<u(t, x)<1$. They showed that the length of this region tends to a stationary distribution which implies that the wavefront does not degenerate. In [7], the authors were interested in the behavior of wave speed $c(\varepsilon)$ as a function of parameter $\varepsilon$. They proved that for large $\varepsilon, c(\varepsilon)$ satisfies the inequality $\liminf _{\varepsilon \rightarrow \infty} \varepsilon^{2} c(\varepsilon) \geq 2$. For small $\varepsilon \leq 1 / 10$, it satisfies the inequality $2-N \ln \ln (1 / \varepsilon) /(\ln \varepsilon)^{2} \leq c(\varepsilon) \leq 2$, where $N$ is a universal constant. In [30], the authors proved that the speed of wavefronts for small $\varepsilon$ is $2-\pi^{2}\left|\ln \varepsilon^{2}\right|^{-2}$ with an error of order $(\ln |\ln \varepsilon|)|\ln \varepsilon|^{-3}$.

In [38], from a point of random dynamical system, the authors considered the reaction-diffusion equation in random media

$$
\frac{\partial}{\partial t} u=u_{x x}+F\left(\theta_{t, x} \omega, u\right),
$$

including time and/or space recurrent, almost periodic, quasi-periodic, periodic ones as special cases, where $\omega \in \Omega$. $(\Omega, \mathcal{F}, \mathbb{P})$ is a probability space. $\left(\Omega, \mathcal{F}, \mathbb{P},\left\{\theta_{t, x}\right\}_{t, x \in \mathbf{R}}\right)$ is a metric dynamical system. $F: \Omega \times \mathbf{R} \rightarrow \mathbf{R}$ is measurable. For each $\omega \in \Omega, F\left(\theta_{t, x} \omega, u\right)$ is Hölder continuous in $t, x$ and Lipschitz continuous in $u$. The authors extended the classical notion of traveling wave solution to traveling wave solution in general random media and adopted a point of view that random traveling wave solutions are limits of certain wavelike solutions. A general theory of existence of random traveling wave solution was established. It shows that the existence of a wave-like solution implies the existence of a critical traveling wave solution, which is the random traveling wave solution with minimal propagating speed.

In $[10,12,34,35]$, the authors considered a general form of KPP equation perturbed by environmental noise, with Heaviside initial condition,

$$
\left\{\begin{array}{l}
d u(t, x)=\left(\frac{D}{2} u_{x x}(t, x)+u(t, x) c(u(t, x))\right) d t+k(t) u(t, x) d W_{t}, \\
u(0, x)=\chi_{(-\infty, l]}(x),
\end{array}\right.
$$

where $c: \mathbf{R}^{+} \rightarrow \mathbf{R}$. $l$ may be a constant or a random variable. Under the assumption that $c \in C^{1}\left(\mathbf{R}^{+}\right)$is strictly decreasing, $c_{0}=c(0)>0$, there 
is $\theta_{0}>0$ such that $c(\theta) \leq 0$ for $\theta \geq \theta_{0}$, and $k$ is not identically zero, the traveling wave solution to (1.9) was studied in [12] by using HamiltonJacobi theory and some computer simulations of behavior of solutions were produced when $l$ and $k$ are deterministic. The noise is called a strong noise if $\liminf _{t \rightarrow \infty}(1 / 2 t) \int_{0}^{t} k^{2}(s) d s>c_{0}$. The noise is weak if $\int_{0}^{\infty} k^{2}(s) d s<\infty$. When the noise is neither strong nor weak, the noise is called a moderately strong noise. The authors showed that the asymptotic behavior of the solution depends on the strength of the noise. If the noise is strong, the solution tends to zero. If the noise is moderately strong, the solution may tend to a traveling wave solution or may be destroyed. The solution tends to the same traveling wave solution as the solution of the deterministic equation if the noise is weak. In [34], the authors considered the similar problems but with either $l$ or $k$ being random. Suppose the limit $k_{\infty}:=\lim _{t \rightarrow \infty}(1 / 2 t) \int_{0}^{t} k^{2}(s) d s$ exists. The wavefront for large time is known as $x=\sqrt{D\left(2 c_{0}-2 k_{\infty}\right)} t$. There are constants $c_{1}$, $c_{2}, c_{3}>0$ such that when $x>\left(\sqrt{D\left(2 c_{0}-2 k_{\infty}\right)}+h\right) t$,

$$
u(t, x)<\exp \left(-c_{1} t\right) \text { a.s. }
$$

and when $x<\left(\sqrt{D\left(2 c_{0}-2 k_{\infty}\right)}-h\right) t$,

$$
\exp \left(-c_{3} \sqrt{2 t \ln \ln t}\right) \leq u(t, x) \leq \exp \left(c_{2} \sqrt{2 t \ln \ln t}\right) \text { a.s. }
$$

for any $h>0$ and sufficiently large $t[10,12,34]$. That is, ahead of the front, the solution to (1.9) is exponentially small almost surly. But behind the front, the solution to (1.9) is oscillatory. In [35], the authors obtained the ergodicity and the pathwise property of the solution to (1.9). They let $c(u)=c_{4}(1-u)$ and $k$ be a constant. Then the front becomes $x=\sqrt{D\left(2 c_{4}-k^{2}\right)} t$. The authors showed the ergodicity by

$$
\begin{aligned}
\lim _{t \rightarrow \infty} \frac{1}{t} \int_{0}^{t} \frac{\inf }{x \leq\left(\sqrt{D\left(2 c_{4}-k^{2}\right)}-h\right) t} u(s, x) d s \\
\quad=\lim _{t \rightarrow \infty} \frac{1}{t} \int_{0}^{t} \sup _{x} u(s, x) d s \\
\quad=1-\frac{k^{2}}{2 c_{4}} \text { a.s. }
\end{aligned}
$$

and

$$
\lim _{t \rightarrow \infty} \frac{1}{t} \int_{0}^{t} \sup _{x \geq\left(\sqrt{D\left(2 c_{4}-k^{2}\right)}+h\right) t} u(s, x) d s=0 \text { a.s. }
$$

for any $h>0$. For the pathwise property of the solution, they showed that

$$
\lim _{t \rightarrow \infty}\left|u(t, x, \omega)-Z\left(\theta_{t} \omega\right)\right|=0 \text { a.s. }
$$

for any $h>0$ and $x<\left(\sqrt{D\left(2 c_{4}-k^{2}\right)}-h\right) t$, where $\theta_{t}$ is the canonical Brownian shift on a probability space $(\Omega, \mathcal{F}, \mathbb{P})$ and

$$
Z(\omega)=\left(c_{4} \int_{0}^{\infty} \exp \left(c_{4} s-\frac{1}{2} k^{2} s+k W_{s}\right) d s\right)^{-1} .
$$


The authors in $[10,12,34,35]$ focused on the profile of the traveling wave solution for large time.

Basing on the results in $[10,12,34,35]$, in this paper, we consider stochastic traveling wave solution to (1.9) under the sense as [39], that is, choosing a suitable wavefront $R(t)$ and proving that $u(t, \cdot+R(t))$ is a stationary process and $\lim _{t \rightarrow \infty} R(t) / t$ exists almost surely. We pay more attention to the law of traveling wave solution rather than the profile of it, which is different from $[10,12,34,35]$. Although the solution to (1.9) does not satisfy the property of compact support. But the property of exponentially decay with respect to spacial variable is sufficient to obtain the result, which we will show in the following. In Sect. 2, some preliminaries are given which are foundations to continue the following works. In Sect. 3, under the assumption that the solution to (1.9) starting at $u_{0}(x)=\chi_{(-\infty, 0]}(x)$ survives forever, defining a suitable marker of wavefront $R(t)$, we prove that $u(t, \cdot+R(t))$ is a stationary process so that the existence of stochastic traveling wave solution to (1.9) will be verified. In Sect. 4, we consider the asymptotic property of the wavefront $R(t)$. We show that $\lim _{t \rightarrow \infty} R(t) / t$ exists almost surely and coincides with the front obtained in [34], which states that the result we obtain is uniform with the result in [34].

We mention that traveling wave solutions to some important water wave equations perturbed by noise were also investigated. In the earlier paper [42], the authors studied the stochastic $\mathrm{KdV}$ equation

$$
u_{t}+6 u u_{x}+u_{x x x}=\zeta_{t} .
$$

They obtained an explicit random soliton solution

$$
u(t, x)=2 \eta^{2} \operatorname{sech}^{2}\left(\eta\left(x-4 \eta^{2} t-x_{0}-6 \int_{0}^{t} W_{s} d s\right)\right)+W_{t}
$$

and analyzed the asymptotic behavior of expectation of this soliton solution (1.17). Later another paper [16] considered a stochastic, damped KdV equation

$$
u_{t}+6 u u_{x}+u_{x x x}=\varepsilon \zeta_{t, x} R[u]-\varepsilon \gamma u .
$$

The authors applied singular perturbation theory to the study of soliton of (1.18), which is influenced by space and time dependent external noise. Since then, some authors made contributions to the study of stochastic versions of $\mathrm{KdV}$ equation, especially the Wick type of the equation [9,43,44]. Some general form of explicit soliton solutions were obtained. They were the natural extensions of classical soliton solutions.

\section{Preliminaries}

Throughout the paper, we let $\Omega:=\mathcal{S}^{\prime}$ be the space of tempered distributions, $\mathcal{F}:=\mathcal{B}$ be the $\sigma$-algebra on $\mathcal{S}^{\prime}$, and $(\Omega, \mathcal{F}, \mathbb{P})$ be the white noise probability space as it is defined in [17]. Denote by $\mathbb{E}$ the expectation with respect to $\mathbb{P}$. Let $W=\left\{W_{t}: 0 \leq t<\infty\right\}$ be the Brownian motion given by the coordinate process, $\zeta_{t}$ be the distributional time derivative of $W_{t}$ and $\mathcal{F}_{t}:=\sigma\left\{W_{s}: 0 \leq\right.$ $s \leq t\}$ be the $\sigma$-algebra flow on $\Omega$. 
Definition 2.1. A stochastic process $u:[0, \infty) \times \mathbf{R} \times \mathcal{S}^{\prime} \rightarrow \mathbf{R}$ is called a strong solution of (1.9) if

(a) $u(\omega) \in C^{0,2}((0, \infty) \times \mathbf{R})$ a.s.,

(b) $u(t, x), u_{x x}(t, x) \in L^{2}(d \mathbb{P})$ for all $(t, x) \in(0, \infty) \times \mathbf{R}$,

(c) $u$ satisfies (1.9) a.s. in the sense that for all $0<t<\infty$ and $x \in \mathbf{R}$,

$$
\begin{aligned}
u(t, x)= & u_{0}(x)+\int_{0}^{t}\left(\frac{D}{2} u_{x x}(s, x)+u(s, x) c(u(s, x))\right) d s \\
& +\int_{0}^{t} k(s) u(s, x) d W_{s} \text { a.s. }
\end{aligned}
$$

Since $u_{0}$ may have discontinuities, $u$ will satisfy the initial condition in the sense that for almost all $x \in \mathbf{R}, \lim _{t \downarrow 0} u(t, x, \omega)=u_{0}(x)$ a.s. Assuming $k \in L_{l o c}^{2}\left(\mathbf{R}^{+}\right)$, the existence and uniqueness of strong solution to (1.9) with piecewise continuous and nonnegative initial condition have been obtained in [34]. The solution enjoys the following properties:

Proposition 2.2. Let $u$ denote the strong solution of (1.9), then

(a) $u(t, x) \geq 0$ for all $(t, x) \in \mathbf{R}^{+} \times \mathbf{R}$ a.s.,

(b) If $x \mapsto u_{0}(x)$ is decreasing a.s., then $x \mapsto u(t, x, \omega)$ is decreasing for each $t \in \mathbf{R}^{+}$and a.e. $\omega \in \mathcal{S}^{\prime}$.

Define

$$
\mathcal{E}_{t}(k):=\exp \left(\int_{0}^{t} k(s) d W_{s}-\frac{1}{2} \int_{0}^{t} k^{2}(s) d s\right), 0 \leq t \leq \infty .
$$

Let $u$ be the solution to (1.9) with initial condition $u_{0}(x)$ and $v(t, x):=$ $u(t, x) \mathcal{E}_{t}^{-1}(k)$. Under the facts

$$
\left(u(t, x) \diamond \mathcal{E}_{\infty}(k)\right)(\omega)=u(t, x, \omega-k) \mathcal{E}_{\infty}(k) \text { a.s. }
$$

and

$$
\frac{d}{d t} \mathcal{E}_{t}(k)=k(t) \zeta_{t} \diamond \mathcal{E}_{t}(k), 0<t<\infty
$$

where $\diamond$ is the Wick product, a straightforward calculation shows that

$$
\frac{\partial}{\partial t} v=\frac{D}{2} v_{x x}+v c\left(v \mathcal{E}_{t}(k)\right), v(0, x, \omega)=u_{0}(x, \omega-k) a . s .
$$

for $(t, x) \in \mathbf{R}^{+} \times \mathbf{R}$. Applying the Feynman-Kac formula to (2.4) gives

$$
\begin{aligned}
v(t, x, \omega)= & \overline{\mathbb{E}}\left[u_{0}\left(x+\sqrt{D} B_{t}, \omega-k\right)\right. \\
& \left.\times \exp \left(\int_{0}^{t} c\left(v\left(t-s, x+\sqrt{D} B_{s}\right) \mathcal{E}_{t-s}(k, \omega)\right) d s\right)\right] \text { a.s. }
\end{aligned}
$$

for $(t, x) \in \mathbf{R}^{+} \times \mathbf{R} . B=\left\{B_{t}: t \geq 0\right\}$ is a Brownian motion defined on an auxiliary probability space, $(\bar{\Omega}, \overline{\mathcal{F}}, \overline{\mathbb{P}})$, and $\overline{\mathbb{E}}$ denotes the expectation with 
respect to $\overline{\mathbb{P}}$. Substituting $v$ with $u$ in $(2.5)$ gives a stochastic Feynman-Kac formula for solution to (1.9),

$$
\begin{aligned}
u(t, x, \omega)= & \overline{\mathbb{E}}\left[u_{0}\left(x+\sqrt{D} B_{t}, \omega-k\right)\right. \\
& \times \exp \left(\int_{0}^{t} c\left(u\left(t-s, x+\sqrt{D} B_{s}\right)\right) d s\right) \\
& \left.\times \exp \left(\int_{0}^{t} k(s) d W_{s}-\frac{1}{2} \int_{0}^{t} k^{2}(s) d s\right)\right] \text { a.s. }
\end{aligned}
$$

for $(t, x) \in \mathbf{R}^{+} \times \mathbf{R}$.

In the remainder of this section, some definitions of spaces are given on which we consider the traveling wave solution to (1.9) and the Kolmogorov tightness criterion is stated. Define

For continuous functions $f: \mathbf{R} \rightarrow \mathbf{R}$, let $\|f\|_{\lambda}=\sup _{x \in \mathbf{R}}\left\{\left|f(x) e^{-\lambda|x|}\right|\right\}$.

$$
C_{\lambda}^{+}=\left\{f \geq 0: f \in C(\mathbf{R}) \text { and }\left|f(x) e^{-\lambda|x|}\right| \rightarrow 0 \text { as } x \rightarrow \pm \infty\right\}
$$

and

$$
C_{\text {tem }}^{+}=\cap_{\lambda>0} C_{\lambda}^{+} .
$$

Then $C_{\text {tem }}^{+}$is the space of continuous functions with slower than exponential growth. The space $C_{\lambda}^{+}$has the topology given by the norm $\|\cdot\|_{\lambda}$ and $C_{\text {tem }}^{+}$is given by norm family $\left\{\|\cdot\|_{\lambda}: \lambda>0\right\}$. Let

$$
C_{\mathrm{dec}}^{+}=\left\{f \geq 0:\|f\|_{\lambda}<\infty \text { for some } \lambda<0\right\}
$$

be the space of functions with exponential decay.

Let $\left(C\left((0, \infty), C_{\text {tem }}^{+}\right), \mathcal{U}, \mathcal{U}_{t}, U(t)\right)$ be continuous path space, which is the canonical right continuous filtration with the coordinate variables. Next we state the Arzela Ascoli theorem and Kolmogorov tightness criterion for the spaces $C_{\lambda}^{+}$and $C_{\text {tem. }}^{+}$.

A set $\Sigma \subseteq C_{\lambda}^{+}$is relatively compact if and only if

(a) $\{f: f \in \Sigma\}$ are equicontinuous on compacts,

(b) $\lim _{R \rightarrow \infty} \sup _{f \in \Sigma} \sup _{|x| \geq R}\left|f(x) e^{-\lambda|x|}\right|=0$.

A set $\Sigma \subseteq C_{\text {tem }}^{+}$is (relatively) compact if and only if it is (relatively) compact in $C_{\lambda}^{+}$for all $\lambda>0$. For $C<\infty, \gamma>0, \delta>0, \mu<\lambda$, define

$$
\Sigma(C, \delta, \gamma, \mu)=\left\{f:\left|f(x)-f\left(x^{\prime}\right)\right| \leq C\left|x-x^{\prime}\right|^{\gamma} e^{\mu|x|} \text { for all }\left|x-x^{\prime}\right| \leq \delta\right\} .
$$

Then using the above conditions one can show that $\Sigma(C, \delta, \gamma, \mu) \cap\{f$ : $\left.\int_{\mathbf{R}} f(x) e^{-|x|} d x \leq c\right\}$ is compact in $C_{\lambda}^{+}$where $c$ is a constant. If $\left\{X_{n}(\cdot)\right\}_{n \in \mathbf{N}}$ is a $C_{\lambda}^{+}$valued process with $\left\{\int_{\mathbf{R}} X_{n}(x) e^{-|x|} d x\right\}_{n \in \mathbf{N}}$ tight and with $C_{0}<\infty$, $p>0, \gamma>1, \mu<\lambda$ such that for all $n \geq 1$,

$$
\mathbb{E}\left[\left|X_{n}(x)-X_{n}\left(x^{\prime}\right)\right|^{p}\right] \leq C_{0}\left|x-x^{\prime}\right|^{\gamma} e^{\mu p|x|}
$$

for all $\left|x-x^{\prime}\right| \leq 1, x, x^{\prime} \in \mathbf{R}$, then $\left\{X_{n}\right\}_{n \in \mathbf{N}}$ is tight. Indeed, if (2.11) holds and $\bar{\gamma}<(\gamma-1) / p, \mu<\bar{\mu}<\lambda$, then there exist deterministic constants $C=C(\bar{\gamma}, \bar{\mu})<\infty, \rho=\rho(\gamma, \bar{\gamma}, p)>0$ and random $\delta(\omega)$ such that $X(\omega) \in$ 
$\Sigma(C, \delta, \gamma, \bar{\mu})$ and $\mathbb{E}\left[\delta^{-\rho}\right] \leq C\left(C_{0}, \mu, \bar{\mu}, \gamma, \bar{\gamma}, p\right)<\infty$. Similarly, if $\left\{X_{n}(\cdot, \cdot)\right\}_{n \in \mathbf{N}}$ is a $C\left(\left[t_{1}, t_{2}\right], C_{\lambda}^{+}\right)$valued process, $\left\{\int_{\mathbf{R}} X_{n}(0, x) e^{-|x|} d x\right\}_{n \in \mathbf{N}}$ is tight and there are $C_{0}<\infty, p>0, \gamma>2, \mu<\lambda$ such that for all $n \geq 1$,

$$
\mathbb{E}\left[\left|X_{n}(t, x)-X_{n}\left(t^{\prime}, x^{\prime}\right)\right|^{p}\right] \leq C_{0}\left(\left|x-x^{\prime}\right|^{\gamma}+\left|t-t^{\prime}\right|^{\gamma}\right) e^{\mu p|x|}
$$

for all $\left|x-x^{\prime}\right| \leq 1,\left|t-t^{\prime}\right| \leq 1, t, t^{\prime} \in\left[t_{1}, t_{2}\right]$, then $\left\{X_{n}\right\}_{n \in \mathbf{N}}$ is tight. The $C_{\text {tem }}^{+}$ (respectively $C\left((0, \infty), C_{\text {tem }}^{+}\right)$) valued process $\left\{X_{n}\right\}_{n \in \mathbf{N}}$ is tight if and only if it is tight as $C_{\lambda}^{+}$(respectively $C\left((0, \infty), C_{\lambda}^{+}\right)$) valued process for each $\lambda>0$.

\section{Stochastic traveling wave solution}

In this section, we define a marker of wavefront $R(t)$ and prove that $u(t, \cdot+R(t))$ is a stationary process, where $u$ is the solution to (1.9) with initial condition $u_{0}(x)=\chi_{(-\infty, 0]}(x)$. We denote $Q^{f}$ the law of unique solution to (1.9) satisfying $u(0)=f$. A probability measure $\nu$ on $C_{\text {tem }}^{+}$may define a probability measure on $C\left((0, \infty), C_{\text {tem }}^{+}\right)$by

$$
Q^{\nu}(A)=\int_{C_{\mathrm{tem}}^{+}} Q^{f}(A) \nu(d f)
$$

for $A \subseteq C\left((0, \infty), C_{\text {tem }}^{+}\right)$. As in [39], we need to define a marker of wavefront. Since the solution to (1.9) possesses the noncompact support property [31]. So $R_{0}(u(t))$ is no longer a suitable front for traveling wave solution to (1.9). We have to choose another marker of wavefront. Since the solution to (1.9) with Heaviside initial condition is exponentially small almost surly as $x \rightarrow \infty$ $[34,35]$, from the stochastic Feynman-Kac formula (2.6), we may choose

$$
R(f)=\ln \left(\int_{\mathbf{R}} e^{x} f(x) d x\right)
$$

as a suitable marker of wavefront in the following consideration of traveling wave solution to (1.9), which is also a auxiliary front in [39]. Note that $R(f(\cdot+$ $c))=R(f)-c$. On $C\left((0, \infty), C_{\text {tem }}^{+}\right)$, define

$$
R(t)=R(U(t))
$$

and

$$
V(t)=\left\{\begin{array}{l}
0, \quad R(t)=-\infty, \\
U(t, \cdot+R(t)), \quad-\infty<R(t)<+\infty, \\
c_{0}, \quad R(t)=\infty .
\end{array}\right.
$$

Thus $V(t)$ is the wave $U(t)$ shifted so that its wavefront lies at the origin.

Now we summarise the method of proof. Taking the Heaviside initial condition $u_{0}(x)=\chi_{(-\infty, 0]}(x)$, define $\nu_{T}$ the law of $T^{-1} \int_{0}^{T} V(s) d s$ under law $Q^{u_{0}}$. It has been shown in $[12,34]$ that if the strength of the noise is not strong, the solution to (1.9) may survive forever. We suppose that the solution survives forever. We shall show that the sequence $\nu_{T}, T=1,2, \ldots$ is tight and that any limit point is nontrivial. We may check that for any limit point $\nu, Q^{\nu}$ is the law of a traveling wave solution to (1.9). Two ingredients that go into the proof of 
tightness are the Kolmogorov tightness criterion for the unshifted waves and the control on the movement of the wavefront to ensure the shifting of the wave does not destroy the tightness.

First, the next three lemmas give bounds of solution on certain moment needed to check the Kolmogorov tightness criterion.

Lemma 3.1. Suppose that the solution $u$ to (1.9) with initial condition $f \geq 0$ survives forever. Then for $p \in \mathbf{N}, t>0$, there are constants $C_{1}(k, p, t)$ and $C_{2}(k, c)$ such that

$$
Q^{f}\left(U^{p}(t, x)\right) \leq C_{1}(k, p, t)\left(\int_{\mathbf{R}} G(t, x-y) f(y) d y+C_{2}(k, c)\right)^{p}
$$

for $x \in \mathbf{R}$, where $G(t, x)$ is the Green's function of heat equation.

Proof. Denote $w$ solves the following equation

$$
\frac{\partial}{\partial t} w=\frac{D}{2} w_{x x}+w c(w)-\frac{1}{2} k^{2} w, w(0, x)=f(x) .
$$

As in [12], we shall show that

$$
\begin{aligned}
& \exp \left(\inf _{0 \leq \sigma \leq t} \int_{\sigma}^{t} k(s) d W_{s}\right) w(t, x) \\
& \quad \leq u(t, x) \leq \exp \left(\sup _{0 \leq \sigma \leq t} \int_{\sigma}^{t} k(s) d W_{s}\right) w(t, x) \text { a.s. }
\end{aligned}
$$

for $(t, x) \in[0, \infty) \times \mathbf{R}$. To obtain a contradiction, we suppose that there is $\left(t^{\prime}, x^{\prime}\right) \in(0, \infty) \times \mathbf{R}$ such that

$$
u\left(t^{\prime}, x^{\prime}\right)>\exp \left(\sup _{0 \leq \sigma \leq t^{\prime}} \int_{\sigma}^{t^{\prime}} k(s) d W_{s}\right) w\left(t^{\prime}, x^{\prime}\right) .
$$

Then $u\left(t^{\prime}, x^{\prime}\right)>w\left(t^{\prime}, x^{\prime}\right)$. For convenience, we denote $X_{s}^{t^{\prime}}, x^{\prime}=\left(t^{\prime}-s, x^{\prime}+\right.$ $\left.\sqrt{D} B_{s}\right)$ for $s \geq 0$. Define a stopping time

$$
\tau:=\inf \left\{s>0: u\left(X_{s}^{t^{\prime}, x^{\prime}}\right)=w\left(X_{s}^{t^{\prime}, x^{\prime}}\right)\right\}
$$

for each $\omega \in \Omega$. Using (2.6) and by the strong Markov property, we have

$$
\begin{aligned}
u\left(t^{\prime}, x^{\prime}\right)= & \overline{\mathbb{E}}\left[u\left(X_{\tau}^{t^{\prime}, x^{\prime}}\right) \exp \left(\int_{0}^{\tau} c\left(u\left(X_{s}^{t^{\prime}, x^{\prime}}\right)\right) d s\right)\right] \\
& \times \exp \left(\int_{t^{\prime}-\tau}^{t^{\prime}} k(s) d W_{s}-\frac{1}{2} \int_{t^{\prime}-\tau}^{t^{\prime}} k^{2}(s) d s\right) \\
\leq & \overline{\mathbb{E}}\left[w\left(X_{\tau}^{t^{\prime}, x^{\prime}}\right) \exp \left(\int_{0}^{\tau} c\left(w\left(X_{s}^{t^{\prime}, x^{\prime}}\right)\right) d s\right)\right] \\
& \times \exp \left(\int_{t^{\prime}-\tau}^{t^{\prime}} k(s) d W_{s}-\frac{1}{2} \int_{t^{\prime}-\tau}^{t^{\prime}} k^{2}(s) d s\right) \\
\leq & \mathbb{E}\left[w\left(X_{\tau}^{t^{\prime}, x^{\prime}}\right) \exp \left(\int_{0}^{\tau}\left(c\left(w\left(X_{s}^{t^{\prime}, x^{\prime}}\right)\right)-\frac{1}{2} k^{2}\left(t^{\prime}-s\right)\right) d s\right)\right]
\end{aligned}
$$




$$
\begin{aligned}
& \times \exp \left(\sup _{0 \leq \sigma \leq t^{\prime}} \int_{t^{\prime}-\sigma}^{t^{\prime}} k(s) d W_{s}\right) \\
= & \exp \left(\sup _{0 \leq \sigma \leq t^{\prime}} \int_{t^{\prime}-\sigma}^{t^{\prime}} k(s) d W_{s}\right) w\left(t^{\prime}, x^{\prime}\right) \text { a.s. }
\end{aligned}
$$

which contradicts (3.8) and proves the upper bound in (3.7). The lower bound is shown similarly.

Since the property of function $c$, there exist constants $a=a(c)$ and $b=b(c)$, such that $c(x) \leq a-b x$ for $x \in[0, \infty)$. Denote $\bar{w}$ the solution to the following equation

$$
\frac{\partial}{\partial t} \bar{w}=\frac{D}{2} \bar{w}_{x x}+\bar{w}\left(a-\frac{1}{2} k^{2}(t)-b \bar{w}\right), \bar{w}(0, x)=f(x) .
$$

Then by comparison method of parabolic PDE, we have $\bar{w} \geq w$ for $(t, x) \in$ $[0, \infty) \times \mathbf{R}$. Fixing $t>0$, for any $\varepsilon>0$, multiplying $G(t-s+\varepsilon, x-y)$ in (3.11) and integrating, we get

$$
\begin{aligned}
\frac{\partial}{\partial s} \int_{\mathbf{R}} \bar{w}(s, y) G(t-s+\varepsilon, x-y) d y \\
=\left(a-\frac{1}{2} k^{2}(s)\right) \int_{\mathbf{R}} \bar{w}(s, y) G(t-s+\varepsilon, x-y) d y \\
\quad-b \int_{\mathbf{R}} \bar{w}^{2}(s, y) G(t-s+\varepsilon, x-y) d y \\
\leq\left(a-\frac{1}{2} k^{2}(s)\right) \int_{\mathbf{R}} \bar{w}(s, y) G(t-s+\varepsilon, x-y) d y \\
\quad-b\left(\int_{\mathbf{R}} \bar{w}(s, y) G(t-s+\varepsilon, x-y) d y\right)^{2} .
\end{aligned}
$$

Denote $h(s)=\int_{\mathbf{R}} \bar{w}(s, y) G(t-s+\varepsilon, x-y) d y$. Thus (3.12) becomes

$$
\left\{\begin{array}{l}
\frac{d}{d s} h(s) \leq\left(a-\frac{1}{2} \inf _{x \in \mathbf{R}} k^{2}(s)\right) h(s)-b h^{2}(s), \\
h(0)=\int_{\mathbf{R}} f(y) G(t+\varepsilon, x-y) d y .
\end{array}\right.
$$

From the theory of ODE, we get

$$
h(s) \leq \int_{\mathbf{R}} f(y) G(t+\varepsilon, x-y) d y+\frac{a}{b}-\frac{1}{2 b} \inf _{s \in \mathbf{R}} k^{2}(s),
$$

which implies

$$
\int_{\mathbf{R}} \bar{w}(t, y) G(\varepsilon, x-y) d y \leq \int_{\mathbf{R}} f(y) G(t+\varepsilon, x-y) d y+\frac{a}{b}-\frac{1}{2 b} \inf _{t \in \mathbf{R}} k^{2}(t) .
$$

Letting $\varepsilon \rightarrow 0$ in $(3.15)$ gives

$$
\bar{w}(t, x) \leq \int_{\mathbf{R}} f(y) G(t, x-y) d y+\frac{a}{b}-\frac{1}{2 b} \inf _{t \in \mathbf{R}} k^{2}(t) .
$$


Then together with (3.7) and (3.16), we get

$$
\begin{aligned}
u^{p}(t, x) \leq & \exp \left(p \sup _{0 \leq \sigma \leq t} \int_{\sigma}^{t} k(s) d W_{s}\right) \\
& \times\left(\int_{\mathbf{R}} f(y) G(t, x-y) d y+\frac{a}{b}-\frac{1}{2 b} \inf _{t \in \mathbf{R}} k^{2}(t)\right)^{p} \text { a.s. }
\end{aligned}
$$

Taking the expectation in (3.17) gives

$$
Q^{f}\left(U^{p}(t, x)\right) \leq C_{1}(k, p, t)\left(\int_{\mathbf{R}} G(t, x-y) f(y) d y+C_{2}(k, t, c)\right)^{p},
$$

where

$$
\begin{aligned}
C_{1}(k, p, t) & =\mathbb{E}\left[\exp \left(p \sup _{0 \leq \sigma \leq t} \int_{\sigma}^{t} k(s) d W_{s}\right)\right], \\
C_{2}(k, c) & =\frac{a}{b}-\frac{1}{2 b} \inf _{t \in \mathbf{R}} k^{2}(t),
\end{aligned}
$$

which completes the proof of the lemma.

Lemma 3.2. Suppose that the solution $u$ to (1.9) with initial condition $f \geq 0$ survives forever and $\varphi$ has two continuous derivatives with $\varphi, \varphi_{x x} \in C_{\mathrm{dec}}^{+}$such that $\alpha:=\sup \left\{\varphi_{x x}(x) / \varphi(x): x \in \mathbf{R}\right\}<\infty$. Set $\beta:=\int_{\mathbf{R}} \varphi(x) d x$. Then for all $p \geq 2, t>0$, there is a constant $C_{3}(k, p, t, c)$ such that

$$
Q^{f}\left(\left(\int_{\mathbf{R}} U(t, x) \varphi(x) d x\right)^{p}\right) \leq C_{3}(k, p, t, c) .
$$

Proof. Similarly with Lemma 3.1, there are constants $a=a(c)$ and $b=b(c)$ such that $c(x) \leq a-b x$ for $x \in[0, \infty)$. Denote $\bar{u}$ the solution to the following equation

$$
\frac{\partial}{\partial t} \bar{u}=\bar{u}_{x x}+\bar{u}(a-b \bar{u})+k \bar{u} \zeta_{t}, \bar{u}(0, x)=f(x) .
$$

By the comparison method for SPDE in [19], we have $\bar{u} \geq u$ a.s. for $(t, x) \in$ $[0, \infty) \times \mathbf{R}$. Multiplying $\varphi$ in (3.22) and integrating, we get

$$
\begin{aligned}
\int_{\mathbf{R}} \bar{u}(t, x) \varphi(x) d x= & \int_{\mathbf{R}} f(x) \varphi(x) d x+\int_{0}^{t} \int_{\mathbf{R}} \bar{u}(s, x) \varphi_{x x}(x) d x d s \\
& +a \int_{0}^{t} \int_{\mathbf{R}} \bar{u}(s . x) \varphi(x) d x d s-b \int_{0}^{t} \int_{\mathbf{R}} \bar{u}^{2}(s, x) \varphi(x) d x d s \\
& +\int_{0}^{t} \int_{\mathbf{R}} k(s) \bar{u}(s, x) \varphi(x) d x d W_{s} .
\end{aligned}
$$

By Itô's formula, together with (3.23), we get

$$
\begin{aligned}
d\left(\int_{\mathbf{R}} \bar{u}(t, x) \varphi(x) d x\right)^{p}= & p\left(\int_{\mathbf{R}} \bar{u}(t, x) \varphi(x) d x\right)^{p-1}\left(\int_{\mathbf{R}} \bar{u}(t, x) \varphi_{x x}(x) d x\right) d t \\
& -b p\left(\int_{\mathbf{R}} \bar{u}(t, x) \varphi(x) d x\right)^{p-1}\left(\int_{\mathbf{R}} \bar{u}^{2}(t, x) \varphi(x) d x\right) d t
\end{aligned}
$$




$$
\begin{aligned}
& +\left(a p+\frac{1}{2} p(p-1) k^{2}(t)\right)\left(\int_{\mathbf{R}} \bar{u}(t, x) \varphi(x) d x\right)^{p} d t \\
& +p k(t)\left(\int_{\mathbf{R}} \bar{u}(t, x) \varphi(x) d x\right)^{p} d W_{t} .
\end{aligned}
$$

Under the assumption in the lemma, we have the following inequalities

$$
\begin{aligned}
\alpha \mathbb{E} & {\left[\left(\int_{\mathbf{R}} \bar{u}(t, x) \varphi(x) d x\right)^{p}\right] } \\
& \geq \mathbb{E}\left[\left(\int_{\mathbf{R}} \bar{u}(t, x) \varphi(x) d x\right)^{p-1}\left(\int_{\mathbf{R}} \bar{u}(t, x) \varphi_{x x}(x) d x\right)\right]
\end{aligned}
$$

and

$$
\int_{\mathbf{R}} \bar{u}^{2}(t, x) \varphi(x) d x \geq \frac{1}{\beta}\left(\int_{\mathbf{R}} \bar{u}(t, x) \varphi(x) d x\right)^{2} .
$$

(3.26) implies

$$
\begin{aligned}
& \frac{1}{\beta} \mathbb{E}\left[\left(\int_{\mathbf{R}} \bar{u}(t, x) \varphi(x) d x\right)^{p}\right]^{1+1 / p} \\
& \quad \leq \mathbb{E}\left[\left(\int_{\mathbf{R}} \bar{u}(t, x) \varphi(x) d x\right)^{p-1}\left(\int_{\mathbf{R}} \bar{u}^{2}(t, x) \varphi(x) d x\right)\right] .
\end{aligned}
$$

Denote by $g(t)=\mathbb{E}\left[\left(\int_{\mathbf{R}} \bar{u}(t, x) \varphi(x) d x\right)^{p}\right]$. Taking expectation in (3.24), together with (3.25) and (3.27), gives that $g(t)$ is continuously differentiable and satisfies

$$
\frac{d}{d t} g(t) \leq\left(\alpha p+a p+\frac{1}{2} p(p-1) k^{2}(t)\right) g(t)-\frac{b p}{\beta} g^{1+1 / p}(t) .
$$

Define $\kappa(y)=\inf \{t \geq 0: g(t) \leq y\}$. For $t>\kappa\left(\left(2 \beta\left(\alpha+a+(p-1) k^{2}(t) / 2\right) / b\right)^{p}\right)$, we get

$$
g(t)<2 \beta\left(\alpha+a-\frac{1}{2} k^{2}(t)\right)^{p} \frac{1}{b^{p}} .
$$

For $t \leq \kappa\left(\left(2 \beta\left(\alpha+a+(n-1) k^{2}(t) / 2\right) / b\right)^{p}\right)$, we have

$$
\frac{d}{d t} g(t) \leq-\frac{b p}{2 \beta} g^{1+1 / p}(t)
$$

which implies

$$
g(t) \leq\left(\frac{2 \beta}{b}\right)^{p} \frac{1}{t^{p}} .
$$

Associating with (3.29) and (3.31) gives

$$
Q^{f}\left(\left(\int_{\mathbf{R}} U(t, x) \varphi(x) d x\right)^{p}\right) \leq g(t) \leq C_{3}(k, p, t, c),
$$

where

$$
C_{3}(k, p, t, c)=\left(2 \beta\left(\alpha+a-\frac{1}{2} k^{2}(t)\right)^{p} \frac{1}{b^{p}}\right) \vee\left(\left(\frac{2 \beta}{b}\right)^{p} \frac{1}{t^{p}}\right)
$$


Thus we complete the proof of the lemma.

Lemma 3.3. Suppose that the solution u to (1.9) with initial condition $f \geq 0$ survives forever. For $p \geq 2, t>0$, there exists $C_{4}(k, p, t, c)$ such that for all $\left|x-x^{\prime}\right| \leq 1, x, x^{\prime} \in \mathbf{R}$,

$$
Q^{f}\left(\left|U(t, x)-U\left(t, x^{\prime}\right)\right|^{p}\right) \leq C_{4}(k, p, t, c)\left|x-x^{\prime}\right|^{p / 2-1} .
$$

Proof. By the Green's function representation of solution to (1.9), letting $x^{\prime}>$ $x,\left|x-x^{\prime}\right| \leq 1$, we get

$$
\begin{aligned}
& \mathbb{E}\left[\left|u(s, x)-u\left(s, x^{\prime}\right)\right|^{p}\right] \\
& \leq 3^{p-1}\left|\int_{\mathbf{R}}\left(G(s, x-y)-G\left(s, x^{\prime}-y\right)\right) f(y) d y\right|^{p} \\
& \quad+3^{p-1} \mathbb{E}\left[\left|\int_{0}^{s} \int_{\mathbf{R}}\left(G(s-r, x-y)-G\left(s-r, x^{\prime}-y\right)\right) c(u(r, y)) u(r, y) d y d r\right|^{p}\right] \\
& \quad+3^{p-1} \mathbb{E}\left[\left|\int_{0}^{s} \int_{\mathbf{R}}\left(G(s-r, x-y)-G\left(s-r, x^{\prime}-y\right)\right) k(r) u(r, y) d y d W_{r}\right|^{p}\right] .
\end{aligned}
$$

Later we will use the bounds

$$
\int_{0}^{s} \int_{\mathbf{R}}\left|G(s-r, x-y)-G\left(s-r, x^{\prime}-y\right)\right| d y d r \leq C(s)\left|x-x^{\prime}\right|^{1 / 2}
$$

for $\left|x-x^{\prime}\right| \leq 1$. Then using Burkholder's inequality, we bound the third term in (3.35) by

$$
\begin{aligned}
\mathbb{E} & {\left[\left|\int_{0}^{s} \int_{\mathbf{R}}\left(G(s-r, x-y)-G\left(s-r, x^{\prime}-y\right)\right) k(r) u(r, y) d y d W_{r}\right|^{p}\right] } \\
\leq & C(p) \mathbb{E}\left[\left|\int_{0}^{s}\left(\int_{\mathbf{R}}\left|G(s-r, x-y)-G\left(s-r, x^{\prime}-y\right)\right| k(r) u(r, y) d y\right)^{2} d r\right|^{p / 2}\right] \\
\leq & C(p) \mathbb{E}\left[\left|\int_{0}^{s} \int_{\mathbf{R}}\right| G(s-r, x-y)-G\left(s-r, x^{\prime}-y\right)\left|k^{2}(r) u^{2}(r, y) d y d r\right|^{p / 2}\right] \\
\leq & C(p)\left(\int_{0}^{s} \int_{\mathbf{R}}\left|G(s-r, x-y)-G\left(s-r, x^{\prime}-y\right)\right| d y d r\right)^{p / 2-1} \\
& \times \int_{0}^{s} \int_{\mathbf{R}}\left|G(s-r, x-y)-G\left(s-r, x^{\prime}-y\right)\right| k^{p}(r) \mathbb{E}\left[u^{p}(r, y)\right] d y d r
\end{aligned}
$$




$$
\begin{aligned}
\leq & C(p, s)\left|x-x^{\prime}\right|^{p / 2-1} \\
& \times \int_{0}^{s} \int_{\mathbf{R}}\left|G(s-r, x-y)-G\left(s-r, x^{\prime}-y\right)\right| k^{p}(r) \mathbb{E}\left[u^{p}(r, y)\right] d y d r .
\end{aligned}
$$

Then we bound the second term in (3.35) by

$$
\begin{aligned}
\mathbb{E} & {\left[\left|\int_{0}^{s} \int_{\mathbf{R}}\left(G(s-r, x-y)-G\left(s-r, x^{\prime}-y\right)\right) c(u(r, y)) u(r, y) d y d r\right|^{p}\right] } \\
\leq & \mathbb{E}\left[\left|\int_{0}^{s} \int_{\mathbf{R}}\left(G(s-r, x-y)-G\left(s-r, x^{\prime}-y\right)\right) c_{0} u(r, y) d y d r\right|^{p}\right] \\
\leq & C(p, s, c)\left|x-x^{\prime}\right|^{p / 2-1} \\
& \times \int_{0}^{s} \int_{\mathbf{R}}\left|G(s-r, x-y)-G\left(s-r, x^{\prime}-y\right)\right| \mathbb{E}\left[u^{p}(r, y)\right] d y d r .
\end{aligned}
$$

The first term in (3.35) is bounded by

$$
\begin{aligned}
& \left|\int_{\mathbf{R}}\left(G(s, x-y)-G\left(s, x^{\prime}-y\right)\right) f(y) d y\right|^{p} \\
& \quad=\left|\int_{\mathbf{R}} \int_{x}^{x^{\prime}} \frac{(y-z)}{2 s \sqrt{4 \pi s}} e^{-(y-z)^{2} / 4 s} f(y) d z d y\right|^{p} \\
& \quad \leq C^{\prime}(p, s)\left(\int_{\mathbf{R}} \int_{x}^{x^{\prime}} \frac{1}{\sqrt{s}} e^{-(y-z)^{2} / 5 s} f(y) d z d y\right)^{p} \\
& \quad \leq C^{\prime}(p, s)\left|x-x^{\prime}\right|^{p} \int_{\mathbf{R}} \frac{1}{\sqrt{s}} e^{-(y-x)^{2} / 5 s} f^{p}(y) d y .
\end{aligned}
$$

Fix $t, p, t$ as in the lemma and set $s=t / 5$. Applying the Markov property at time $4 s$ and the above estimates imply that there are constants $C_{5}(k, p, t, c)$ and $C_{6}(k, p, t, c)$ such that

$$
\begin{aligned}
& \mathbb{E}\left[\left|u(t, x)-u\left(t, x^{\prime}\right)\right|^{p}\right] \\
& \leq \quad C_{5}(k, p, t, c)\left|x-x^{\prime}\right|^{p / 2-1} \int_{\mathbf{R}} \frac{1}{\sqrt{s}} e^{-(y-x)^{2} / 5 s} \mathbb{E}\left[u^{p}(4 s, y)\right] d y \\
& \quad+C_{6}(k, p, t, c)\left|x-x^{\prime}\right|^{p / 2-1} \\
& \quad \times \int_{4 s}^{t} \int_{\mathbf{R}}\left|G(t-r, x-y)-G\left(t-r, x^{\prime}-y\right)\right| \mathbb{E}\left[u^{p}(r, y)\right] d y d r .
\end{aligned}
$$

From Lemma 3.1, we have

$$
\begin{aligned}
& Q^{f}\left(U(4 s+r, x)^{p}\right) \\
& \quad \leq C_{1}(k, p, r) Q^{f}\left(\left(\int_{\mathbf{R}} U(4 s, y) G(r, x-y) d y+C_{2}(k, c)\right)^{p}\right) \\
& \quad=C_{1}(k, p, r) \sum_{i=0}^{p} \frac{p !}{i !(p-i) !} C_{2}^{p-i}(k, c) Q^{f}\left(\left(\int_{\mathbf{R}} U(4 s, y) G(r, x-y) d y\right)^{i}\right)
\end{aligned}
$$


for $r \in[0, s], x \in \mathbf{R}$. Apply Lemma 3.2 with $\varphi(y)=e^{-\left(1+(x-y)^{2}\right)^{1 / 2}}$ for which $\alpha \leq 3, \beta \leq 2$ and note that $G(r, x-y) \leq C^{\prime}(s) \varphi(y)$. This gives that

$$
Q^{f}\left(\left(\int_{\mathbf{R}} U(4 s, y) G(r, x-y) d y\right)^{i}\right) \leq C_{3}^{\prime}(k, i, 4 s, c),
$$

where

$$
C_{3}^{\prime}(k, i, 4 s, c)=C^{\prime}(s)\left(4\left(3+a-\frac{1}{2} k^{2}(4 s)\right)^{i} \frac{1}{b^{i}}\right) \vee\left(\left(\frac{4}{b}\right)^{i} \frac{1}{(4 s)^{i}}\right) .
$$

Associating with (3.40)-(3.42), we get

$$
Q^{f}\left(\left|U(t, x)-U\left(t, x^{\prime}\right)\right|^{p}\right) \leq C_{4}(k, p, t, c)\left|x-x^{\prime}\right|^{p / 2-1},
$$

where

$$
\begin{aligned}
& C_{4}(k, p, t, c) \\
&= C_{5}(k, p, t, c) \int_{\mathbf{R}} \frac{\sqrt{5}}{\sqrt{t}} e^{-y^{2} / t} d y \sum_{i=0}^{p} \frac{p !}{i !(p-i) !} C_{2}^{p-i}(k, c) C_{3}^{\prime}\left(k, i, \frac{4 t}{5}, c\right) \\
&+2 C_{6}(k, p, t, c) \\
& \times \int_{0}^{\frac{t}{5}} \int_{\mathbf{R}} G\left(\frac{t}{5}-r, y\right) \sum_{i=0}^{p} \frac{p !}{i !(p-i) !} C_{2}^{p-i}(k, c) C_{3}^{\prime}\left(k, i, \frac{4 t}{5}, c\right) d y d r
\end{aligned}
$$

which completes the proof of the lemma.

Under the assumption that the solution to (1.9) with Heaviside initial condition survives forever, now we tend to control the wavefront $R(t)$. The following two lemmas give some estimations on the movement of the wavefront ensuring the shifting of the wave dose not destroy the tightness.

Lemma 3.4. Suppose that the solution $u$ to (1.9) with initial condition $u_{0}(x)=$ $\chi_{(-\infty, 0]}(x)$ survives forever. Then there exists a constant $C<\infty$ such that

$$
Q^{u_{0}}(R(t)) \geq-C(1+t)
$$

Proof. Let $\psi_{0}(x)=\chi_{(-1,1)}(x)$. Define $\psi_{r}(x)=\psi_{0}(x+r)$ where $r \geq 2$. Then $u_{0} \geq \sum_{j=1}^{\infty} \psi_{j r}$. The comparison method for SPDE implies that $Q^{u_{0}}(R(t)) \geq$ $Q^{\sum_{j=1}^{\infty} \psi_{j r}}(R(t))$. Consider the following equations

$$
\left\{\begin{array}{l}
\frac{\partial}{\partial t} u^{(j)}=\frac{D}{2} u_{x x}^{(j)}+c\left(u^{(j)}\right) u^{(j)}+k(t) u^{(j)} \zeta_{t} \\
u^{(j)}(0, x)=\psi_{j r}(x), \\
j=1,2, \ldots
\end{array}\right.
$$

Next we may couple the solution $u$ to (1.9) starting at $u_{0}$ with a sequence of independent processes $u^{(j)}$ solving (3.47). Denote $u^{(1,2)}$ the solution to (1.9) with initial condition $\psi_{r}+\psi_{2 r}$. It is easy to see that $u^{(1)} \leq u^{(1,2)}, u^{(2)} \leq$ 
$u^{(1,2)}$ a.s. by comparison method. By the stochastic Feynman-Kac formula, we get

$$
\begin{aligned}
u^{(1,2)}(t, x)= & \overline{\mathbb{E}}\left[\psi_{r}\left(x+\sqrt{D} B_{t}\right)+\psi_{2 r}\left(x+\sqrt{D} B_{t}\right)\right. \\
& \times \exp \left(\int_{0}^{t} c\left(u^{(1,2)}\left(t-s, x+\sqrt{D} B_{s}\right)\right) d s\right) \\
& \left.\times \exp \left(\int_{0}^{t} k(s) d W_{s}-\frac{1}{2} \int_{0}^{t} k^{2}(s) d s\right)\right] d s \\
\leq & \overline{\mathbb{E}}\left[\psi_{r}\left(x+\sqrt{D} B_{t}\right)\right. \\
& \times \exp \left(\int_{0}^{t} c\left(u^{(1)}\left(t-s, x+\sqrt{D} B_{s}\right)\right) d s\right) \\
& \left.\times \exp \left(\int_{0}^{t} k(s) d W_{s}-\frac{1}{2} \int_{0}^{t} k^{2}(s) d s\right)\right] d s \\
& +\overline{\mathbb{E}}\left[\psi_{2 r}\left(x+\sqrt{D} B_{t}\right)\right. \\
& \times \exp \left(\int_{0}^{t} c\left(u^{(2)}\left(t-s, x+\sqrt{D} B_{s}\right)\right) d s\right) \\
& \left.\times \exp \left(\int_{0}^{t} k(s) d W_{s}-\frac{1}{2} \int_{0}^{t} k^{2}(s) d s\right)\right] d s \\
= & u^{(1)}(t, x)+u^{(2)}(t, x) a . s .
\end{aligned}
$$

for $(t, x) \in[0, \infty) \times \mathbf{R}$. By (2.6) and Doob's inequality, we get

$$
\begin{aligned}
& \mathbb{E}\left[\int_{-\infty}^{-\frac{3}{2} r} u^{(1)}(t, x) e^{x} d x\right] \\
& \quad \leq e^{c_{0} t} \int_{-\infty}^{-\frac{3}{2} r} \overline{\mathbb{P}}\left(\frac{-r-x-1}{\sqrt{D}} \leq B_{t} \leq \frac{-r-x+1}{\sqrt{D}}\right) e^{x} d x \\
& \quad \leq e^{c_{0} t} \int_{-\infty}^{-\frac{3}{2} r} \exp \left(x-\frac{(x+r-1)^{2}}{2 D t}\right) d x \\
& \quad \leq \frac{\sqrt{2 D t}}{2} \exp \left(c_{0} t+\frac{9}{2} D t-3-\frac{(r-2+6 D t)^{2}}{8 D t}\right) .
\end{aligned}
$$

Therefore for any $\varepsilon>0$, we can choose suitable constants $C_{1}, C_{2}$ and set $r=C_{1} t+C_{2}$, such that

$$
\mathbb{E}\left[\int_{-\infty}^{-\frac{3}{2} r} u^{(1)}(t, x) e^{x} d x\right]<\frac{\varepsilon}{4} .
$$


A similar computation gives that

$$
\mathbb{E}\left[\int_{-\frac{3}{2} r}^{\infty} u^{(2)}(t, x) e^{x} d x\right] \leq \frac{\sqrt{2 D t}}{2} \exp \left(c_{0} t+\frac{9}{2} D t-3-\frac{(r-2+6 D t)^{2}}{8 D t}\right) .
$$

Then the choice of $r$ implies

$$
\mathbb{E}\left[\int_{-\frac{3}{2} r}^{\infty} u^{(2)}(t, x) e^{x} d x\right]<\frac{\varepsilon}{4}
$$

Thus, together with (3.50) and (3.52), we have

$$
\begin{aligned}
\mathbb{E} & {\left[\left|\int_{\mathbf{R}} u^{(1,2)}(t, x) e^{x} d x-\int_{\mathbf{R}} u^{(1)}(t, x) e^{x} d x-\int_{\mathbf{R}} u^{(2)}(t, x) e^{x} d x\right|\right] } \\
\leq & \mathbb{E}\left[\left|\int_{-\infty}^{-\frac{3}{2} r} u^{(1,2)}(t, x) e^{x} d x-\int_{-\infty}^{-\frac{3}{2} r} u^{(1)}(t, x) e^{x} d x-\int_{-\infty}^{-\frac{3}{2} r} u^{(2)}(t, x) e^{x} d x\right|\right] \\
& +\mathbb{E}\left[\left|\int_{-\frac{3}{2} r}^{\infty} u^{(1,2)}(t, x) e^{x} d x-\int_{-\frac{3}{2} r}^{\infty} u^{(1)}(t, x) e^{x} d x-\int_{-\frac{3}{2} r}^{\infty} u^{(2)}(t, x) e^{x} d x\right|\right] \\
\leq & \mathbb{E}\left[\left|\int_{-\infty}^{-\frac{3}{2} r} u^{(1,2)}(t, x) e^{x} d x-\int_{-\infty}^{-\frac{3}{2} r} u^{(2)}(t, x) e^{x} d x\right|\right] \\
& +\mathbb{E}\left[\left|\int_{-\frac{3}{2} r}^{\infty} u^{(1,2)}(t, x) e^{x} d x-\int_{-\frac{3}{2} r}^{\infty} u^{(1)}(t, x) e^{x} d x\right|\right] \\
& +\mathbb{E}\left[\left|\int_{-\frac{3}{2} r}^{\infty} u^{(2)}(t, x) e^{x} d x\right|\right]+\mathbb{E}\left[\left|\int_{-\infty}^{-\frac{3}{2} r} u^{(1)}(t, x) e^{x} d x\right|\right] \\
< & \frac{\varepsilon}{4}+\frac{\varepsilon}{4}+\frac{\varepsilon}{4}+\frac{\varepsilon}{4} \\
= & \varepsilon, \quad
\end{aligned}
$$

which implies

$$
\mathbb{P}\left(\int_{\mathbf{R}} u^{(1,2)}(t, x) e^{x} d x>\int_{\mathbf{R}} u^{(1)}(t, x) e^{x} d x+\int_{\mathbf{R}} u^{(2)}(t, x) e^{x} d x-\varepsilon\right)>1-\varepsilon .
$$

We claim that there exists $\mu>0$ such that

$$
\mathbb{P}\left(\int_{\mathbf{R}} u(t, x) e^{x} d x>\int_{\mathbf{R}} u^{(1,2)}(t, x) e^{x} d x+\mu\right)>\mu .
$$

To see this, we suppose the contrary: for any $\mu>0$, there is $t=t(\mu)$ such that $\mathbb{P}\left(\int_{\mathbf{R}} u(t, x) e^{x} d x>\int_{\mathbf{R}} u^{(1,2)}(t, x) e^{x} d x+\mu\right) \leq \mu$. But we have

$$
\begin{aligned}
1-\mu & <\mathbb{P}\left(0 \leq \int_{\mathbf{R}} u(t, x) e^{x} d x-\int_{\mathbf{R}} u^{(1,2)}(t, x) e^{x} d x \leq \mu\right) \\
& \leq \mathbb{P}\left(0 \leq \int_{-2 r}^{\infty} u(t, x) e^{x} d x-\int_{-2 r}^{\infty} u^{(1,2)}(t, x) e^{x} d x \leq \mu\right)
\end{aligned}
$$




$$
\leq \mathbb{P}\left(0 \leq \int_{-2 r}^{\infty} u(t, x) d x-\int_{-2 r}^{\infty} u^{(1,2)}(t, x) d x \leq \mu e^{2 r}\right) \leq 1 .
$$

That is

$$
\mathbb{P}\left(\int_{-2 r}^{\infty} u(t, x) d x-\int_{-2 r}^{\infty} u^{(1,2)}(t, x) d x \rightarrow 0\right) \rightarrow 1 \text { as } \mu \rightarrow 0,
$$

which implies

$$
\mathbb{P}\left(m\left(\left\{x \in(-\infty,-2 r]: u(t, x)-u^{(1,2)}(t, x)>\varepsilon\right\}\right) \rightarrow 0\right) \rightarrow 1 \text { as } \mu \rightarrow 0 .
$$

This contradicts the decreasing property of $u$ with respect to $x$. Let $\varepsilon=\mu$. Similarly with the above estimations, for some $m \in \mathbf{N}$, denoting $u^{(1, m)}$ the solution to (1.9) with initial condition $\sum_{j=1}^{m} \psi_{j r}$, we get

$$
\mathbb{P}\left(\Omega_{1}\right)>1-\mu
$$

and

$$
\mathbb{P}\left(\Omega_{2}\right)>\mu,
$$

where

$$
\Omega_{1}=\left\{\int_{\mathbf{R}} u^{(1, m)}(t, x) e^{x} d x>\sum_{j=1}^{m} \int_{\mathbf{R}} u^{(j)}(t, x) e^{x} d x-\mu\right\}
$$

and

$$
\Omega_{2}=\left\{\int_{\mathbf{R}} u(t, x) e^{x} d x>\int_{\mathbf{R}} u^{(1, m)}(t, x) e^{x} d x+\mu\right\} .
$$

On the other hand, we claim that there exists $\delta>0$ such that

$$
Q^{\psi_{0}}\left(\int_{\mathbf{R}} U(t, x) d x \geq \delta\right) \geq \delta
$$

To see this, we suppose the contrary: for each $\delta>0$ there exists $t=t(\delta)$ such that $Q^{\psi_{0}}\left(\int_{\mathbf{R}} U(t, x) d x \geq \delta\right)<\delta$. Denote $w_{0}$ the solution to (3.6) with initial condition $\psi_{0}$. We set

$$
\delta<\min \left\{\frac{1}{2}, \inf _{s \in[0, \infty)}\left\{\int_{\mathbf{R}} w_{0}(s, x) d x \exp \left(-2 \mathbb{E}\left[\left|\int_{0}^{s} k(\tau) d W_{\tau}\right|\right]\right)\right\}\right\} .
$$

Then under the assumption, we have

$$
Q^{\psi_{0}}\left(\int_{\mathbf{R}} U(t, x) d x<\delta\right) \geq \frac{1}{2} .
$$

By (3.7) and Doob's inequality, we get

$$
\begin{aligned}
& Q^{\psi_{0}}\left(\int_{\mathbf{R}} U(t, x) d x<\delta\right) \\
& \quad \leq \mathbb{P}\left(\exp \left(\inf _{0 \leq \sigma \leq t} \int_{\sigma}^{t} k(s) d W_{s}\right) \int_{\mathbf{R}} w_{0}(t, x) d x<\delta\right)
\end{aligned}
$$




$$
\begin{aligned}
& =\mathbb{P}\left(\inf _{0 \leq \sigma \leq t} \int_{\sigma}^{t} k(s) d W_{s}<\ln \delta-\ln \left(\int_{\mathbf{R}} w_{0}(t, x) d x\right)\right) \\
& \leq \mathbb{P}\left(\sup _{0 \leq \sigma \leq t}\left|\int_{0}^{\sigma} k(s) d W_{s}\right|>-\ln \delta+\ln \left(\int_{\mathbf{R}} w_{0}(t, x) d x\right)\right) \\
& \leq\left(-\ln \delta+\ln \left(\int_{\mathbf{R}} w_{0}(t, x) d x\right)\right)^{-1} \mathbb{E}\left[\left|\int_{0}^{t} k(s) d W_{s}\right|\right] \\
& <\frac{1}{2},
\end{aligned}
$$

which contradicts (3.65). The property of symmetry gives $Q^{\psi_{0}}\left(\int_{0}^{\infty} U(t, x) d x \geq\right.$ $\delta / 2) \geq \delta / 2$. Therefore, we have

$$
\begin{aligned}
\mathbb{P}\left(R\left(u^{(j)}(t)\right) \geq \frac{1}{a} \ln \frac{\delta}{2}-j r\right) & =\mathbb{P}\left(R\left(u^{(0)}(t)\right) \geq \frac{1}{a} \ln \frac{\delta}{2}\right) \\
& \geq \mathbb{P}\left(\int_{0}^{\infty} u^{(0)}(t, x) d x \geq \frac{\delta}{2}\right) \\
& \geq \frac{\delta}{2} .
\end{aligned}
$$

Then together with (3.59), (3.60) and (3.67), we get

$$
\begin{aligned}
& \mathbb{P}\left(\left\{R(u(t)) \leq \ln \frac{\delta}{2}-m r\right\} \cap \Omega_{1} \cap \Omega_{2}\right) \\
& \quad \leq \mathbb{P}\left(\sum_{j=1}^{m} \int_{\mathbf{R}} u^{(j)}(t, x) e^{x} d x \leq \exp \left(\ln \frac{\delta}{2}-m r\right)\right) \\
& \quad \leq \mathbb{P}\left(R\left(u^{(j)}(t)\right) \leq \ln \frac{\delta}{2}-j r, j=1,2, \ldots, m\right) \\
& \quad \leq\left(1-\frac{\delta}{2}\right)^{m} .
\end{aligned}
$$

That is

$$
\mathbb{P}\left(R(u(t)) \leq \ln \frac{\delta}{2}-m C_{2}-m C_{1} t\right) \leq\left(1-\frac{\delta}{2}\right)^{m} \frac{1}{\mu-\mu^{2}},
$$

which implies the lower bound in the lemma.

Lemma 3.5. Suppose that the solution $u$ to (1.9) with initial condition $u_{0}(x)=$ $\chi_{(-\infty, 0]}(x)$ survives forever. For $t>0$, there exists constant $C^{\prime}<\infty$ such that

$$
Q^{\nu_{T}}(|R(t)| \geq d) \leq \frac{C^{\prime}}{d}
$$

for all $d>0, T \geq 1$.

Proof. Let $v$ be the solution to the following equation starting at $f$,

$$
\frac{\partial}{\partial t} v=\frac{D}{2} v_{x x}+c_{0} v+k(t) v \zeta_{t}, v(0, x)=f(x) \geq 0 .
$$


The Green's function representation of $v$ gives

$v(t, x)=\int_{\mathbf{R}} e^{c_{0} t} G(t, x-y) f(y) d y+\int_{0}^{t} \int_{\mathbf{R}} k(s) e^{c_{0} t} G(t-s, x-y) d y d W_{s}$.

The comparison method for SPDE shows that

$$
\begin{aligned}
Q^{f}\left(\int_{\mathbf{R}} U(t, x) e^{x} d x\right) & \leq \mathbb{E}\left[\int_{\mathbf{R}} v(t, x) e^{x} d x\right] \\
& \leq e^{c_{0} t} \int_{\mathbf{R}} f(y) \int_{\mathbf{R}} \frac{1}{\sqrt{4 \pi t}} e^{-|x-y|^{2} / 4 t} e^{x} d x d y \\
& =e^{c_{0} t} \int_{\mathbf{R}} \frac{1}{\sqrt{4 \pi t}} e^{-|x|^{2} / 4 t} e^{x} d x \int_{\mathbf{R}} f(x) e^{x} d x \\
& =e^{c_{0} t+t} \int_{\mathbf{R}} f(x) e^{x} d x
\end{aligned}
$$

Note that under $Q^{u_{0}}$

$$
\begin{aligned}
\int_{\mathbf{R}} V(t, x) e^{x} d x & =\int_{\mathbf{R}} U(t, x+R(t)) e^{x} d x \\
& =e^{-R(t)} \int_{\mathbf{R}} U(t, x) e^{x} d x \\
& =1 .
\end{aligned}
$$

Associating with (3.73) and (3.74) gives

$$
\begin{aligned}
Q^{\nu_{T}}(R(t) \geq d) & =\frac{1}{T} \int_{0}^{T} Q^{u_{0}}\left(Q^{V(s)}(R(t) \geq d)\right) d s \\
& =\frac{1}{T} \int_{0}^{T} Q^{u_{0}}\left(Q^{V(s)}\left(e^{-d} \int_{\mathbf{R}} U(t, x) e^{x} d x \geq 1\right)\right) d s \\
& \leq e^{-d} \frac{1}{T} \int_{0}^{T} Q^{u_{0}}\left(Q^{V(s)}\left(\int_{\mathbf{R}} U(t, x) e^{x} d x\right)\right) d s \\
& \leq e^{-d+c_{0} t+t} \frac{1}{T} \int_{0}^{T} \int_{\mathbf{R}} V(s, x) e^{x} d x d s \\
& \leq e^{-d+c_{0} t+t},
\end{aligned}
$$

which proves half of the lemma. On the other hand, Jensen's inequality gives

$$
\begin{aligned}
Q^{f}(R(t)) & \leq \frac{1}{a} \ln \left(Q^{f}\left(\int_{\mathbf{R}} U(t, x) e^{x} d x\right)\right) \\
& \leq \ln \left(e^{c_{0} t+t} \int_{\mathbf{R}} f(x) e^{x} d x\right) \\
& \leq c_{0} t+t+R(f) .
\end{aligned}
$$

An estimation shows

$$
\frac{1}{T} Q^{u_{0}}\left(\int_{t}^{T+t} R(s) d s-\int_{0}^{T} R(s) d s\right)
$$




$$
\begin{aligned}
= & \frac{1}{T} Q^{u_{0}}\left(\int_{0}^{T}(R(s+t)-R(s)) d s\right) \\
= & \frac{1}{T} \int_{0}^{T} \int_{\{R(u(s+t))-R(u(s))>-d\}}(R(u(s+t))-R(u(s))) Q^{u_{0}}(d u) d s \\
& +\frac{1}{T} \int_{0}^{T} \int_{\{R(u(s+t))-R(u(s)) \leq-d\}}(R(u(s+t))-R(u(s))) Q^{u_{0}}(d u) d s \\
\leq & \frac{1}{T} \int_{0}^{T} \int_{\{R(u(s+t))-R(u(s))>0\}}(R(u(s+t))-R(u(s))) Q^{u_{0}}(d u) d s \\
& -\frac{d}{T} \int_{0}^{T} Q^{u_{0}}(R(s+t)-R(s) \leq-d) d s \\
\leq & \frac{1}{T} \int_{0}^{T} \int_{0}^{\infty} Q^{u_{0}}(R(s+t)-R(s) \geq y) d y d s \\
& -\frac{d}{T} \int_{0}^{T} Q^{u_{0}}(R(s+t)-R(s) \leq-d) d s \\
= & \int_{0}^{\infty} Q^{\nu_{T}}(R(t) \geq y) d y-d Q^{\nu_{T}}(R(t) \leq-d) .
\end{aligned}
$$

Rearranging (3.77) and applying Lemma 3.4, we get

$$
\begin{aligned}
Q^{\nu_{T}}(R(t) \leq-d) \leq & \frac{1}{d} \int_{0}^{\infty} Q^{\nu_{T}}(R(t) \geq y) d y+\frac{1}{d T} \int_{0}^{T} Q^{u_{0}}(R(s)) d s \\
& -\frac{1}{d T} \int_{t}^{T+t} Q^{u_{0}}(R(s)) d s \\
\leq & \frac{1}{d} \int_{0}^{\infty} e^{-y+c_{0} t+t} d y+\frac{1}{d T} \int_{0}^{T}\left(R\left(u_{0}\right)+c_{0} s+s\right) d s \\
& +\frac{1}{d T} \int_{t}^{T+t} C(1+s) d s \\
\leq & \frac{C^{\prime}}{d}
\end{aligned}
$$

which completes the proof of the lemma.

Associating with Lemma 3.3 and Lemma 3.5, we are sufficient to show the tightness of $\left\{\nu_{T}, T=1,2, \ldots\right\}$.

Lemma 3.6. Suppose that the solution $u$ to (1.9) with initial condition $u_{0}(x)=$ $\chi_{(-\infty, 0]}(x)$ survives forever. Then the sequence $\left\{\nu_{T}, T=1,2, \ldots\right\}$ is tight.

Proof. Recall the set $\Sigma(C, \delta, \gamma, \mu)$ and the condition of tightness stated in the introduction. We have

$$
\begin{aligned}
& \nu_{T}(\Sigma(C, \delta, \gamma, \mu)) \\
& \quad=\frac{1}{T} \int_{0}^{T} Q^{u_{0}}(U(t, \cdot+R(t)) \in \Sigma(C, \delta, \gamma, \mu)) d t
\end{aligned}
$$




$$
\begin{aligned}
& \geq \frac{1}{T} \int_{1}^{T} Q^{u_{0}}\left(U(t, \cdot+R(t-1)) \in \Sigma\left(C e^{-\mu d}, \delta, \gamma, \mu\right),|R(t)-R(t-1)| \leq d\right) \\
& \geq \frac{1}{T} \int_{1}^{T} Q^{u_{0}}\left(Q^{V(t-1)}\left(U(1) \in \Sigma\left(C e^{-\mu d}, \delta, \gamma, \mu\right)\right)\right) d t \\
& -\frac{1}{T} \int_{1}^{T} Q^{u_{0}}(|R(t)-R(t-1)| \geq d) d t \\
& \geq \frac{1}{T} \int_{1}^{T} Q^{u_{0}}\left(Q^{V(t-1)}\left(U(1) \in \Sigma\left(C e^{-\mu d}, \delta, \gamma, \mu\right)\right)\right) d t \\
& -\frac{1}{T} \int_{1}^{T} Q^{u_{0}}\left(Q^{V(t-1)}(|R(1)| \geq d)\right) d t
\end{aligned}
$$

The second term in (3.79) is bounded by $Q^{\nu_{T}}(|R(1)| \geq d) \leq C^{\prime} / d$ from Lemma 3.5. Lemma 3.3 and the criterion of tightness show that given $d, \mu>0$, we can choose $C, \gamma$ and $\delta$ to make the first term in (3.79) as close to $(T-1) / T$ as desired. On the other hand, we have

$$
\nu_{T}\left\{f: \int_{\mathbf{R}} f(x) e^{-|x|} d x \leq \int_{\mathbf{R}} f(x) e^{x} d x=1\right\}=1 .
$$

So given $\mu>0$, we can choose $C, \gamma$ and $\delta$ so that $\nu_{T}(\Sigma(C, \delta, \gamma, \mu) \cap\{f$ : $\left.\left.\int_{\mathbf{R}} f(x) e^{-|x|} d x \leq 1\right\}\right)$ is as close to one as desired for large $T$ and $d$, which completes the proof of the lemma.

By Lemma 3.6 we may take a subsequence $\nu_{T(n)}$ converging to $\nu$. Now we state the main argument that $u(t, \cdot+R(t))$ is a stationary process, which implies that $Q^{\nu}$ is the law of a traveling wave solution to (1.9).

Theorem 3.7. Suppose that the solution $u$ to (1.9) with initial condition $u_{0}(x)=$ $\chi_{(-\infty, 0]}(x)$ survives forever. Then $u(t, \cdot+R(t))$ is a stationary process. $Q^{\nu}$ is the law of a traveling wave solution to (1.9).

Proof. First, we prove the following properties of $\nu$.

$$
\begin{aligned}
& \lim _{T \rightarrow \infty} \nu_{T}\left\{f: \lim _{d \rightarrow \infty} \int_{d}^{\infty} f(x) d x=0\right\}=1 . \\
& \nu\left\{f: \int_{\mathbf{R}} f(x) e^{x} d x=1\right\}=1 . \\
& U(t) \neq 0, \forall t \geq 0, Q^{\nu} \text { a.s. }
\end{aligned}
$$

Under the law $Q^{u_{0}}$, from (2.6), we have

$$
\begin{aligned}
V(t, x)= & \overline{\mathbb{E}}\left[u_{0}\left(x+\sqrt{2} B_{t}\right) \exp \left(\int_{0}^{t} c\left(V\left(t-s, x+\sqrt{2} B_{s}\right)\right) d s\right)\right. \\
& \left.\times \exp \left(-\frac{1}{2} \int_{0}^{t} k^{2}(s) d s+\int_{0}^{t} k(s) d W_{s}\right)\right] \\
\leq & \exp \left(c_{0} t-\frac{1}{2} \int_{0}^{t} k^{2}(s) d s+\int_{0}^{t} k(s) d W_{s}\right) \overline{\mathbb{P}}\left(B_{t} \leq-\frac{x}{\sqrt{2}}\right)
\end{aligned}
$$




$$
\begin{aligned}
= & \exp \left(c_{0} t-\frac{1}{2} \int_{0}^{t} k^{2}(s) d s+\int_{0}^{t} k(s) d W_{s}\right) \\
& \times \frac{1}{\sqrt{2 \pi t}} \int_{-\infty}^{-x / \sqrt{2}} e^{-|y|^{2} / 2 t} d y \text { a.s. }
\end{aligned}
$$

for $t>0$. Again from (2.6), together with (3.84) and Doob's inequality, under the law $Q^{u_{0}}$, we get

$$
\begin{aligned}
U(1, x) \leq & \exp \left(c_{0}-\frac{1}{2} \int_{0}^{1} k^{2}(s) d s+\int_{0}^{1} k(s) d W_{s}\right) \overline{\mathbb{E}}\left[V\left(t-1, x+\sqrt{2} B_{1}\right)\right] \\
\leq & \exp \left(c_{0} t-\frac{1}{2} \int_{0}^{1} k^{2}(s) d s+\int_{0}^{1} k(s) d W_{s}\right) \\
& \times \exp \left(-\frac{1}{2} \int_{0}^{t-1} k^{2}(s) d s+\int_{0}^{t-1} k(s) d W_{s}\right) \\
& \times \frac{1}{2 \pi \sqrt{t-1}} \int_{-\infty}^{+\infty} \int_{-\infty}^{-x / \sqrt{2}-z} e^{-|y|^{2} / 2(t-1)} d y e^{-|z|^{2} / 2} d z \\
= & \exp \left(c_{0} t-\frac{1}{2} \int_{0}^{1} k^{2}(s) d s+\int_{0}^{1} k(s) d W_{s}\right) \\
& \times \exp \left(-\frac{1}{2} \int_{0}^{t-1} k^{2}(s) d s+\int_{0}^{t-1} k(s) d W_{s}\right) \overline{\mathbb{P}}\left(B_{t} \leq-\frac{x}{\sqrt{2}}\right) \\
\leq & \exp \left(c_{0} t-\frac{1}{2} \int_{0}^{1} k^{2}(s) d s+\int_{0}^{1} k(s) d W_{s}\right) \\
& \times \exp \left(-\frac{1}{2} \int_{0}^{t-1} k^{2}(s) d s+\int_{0}^{t-1} k(s) d W_{s}-\frac{x^{2}}{4 t}\right) a . s .
\end{aligned}
$$

Integrating (3.85) with respect to $x$ in $[d, \infty)$ and taking expectation gives

$$
\lim _{d \rightarrow \infty} Q^{u_{0}}\left(Q^{V(t-1)}\left(\int_{d}^{\infty} U(1, x) d x\right)\right) \leq \lim _{d \rightarrow \infty} \sqrt{t} \exp \left(c_{0} t-\frac{d^{2}}{4 t}\right)=0,
$$

which implies that

$$
Q^{u_{0}}\left(Q^{V(t-1)}\left(\lim _{d \rightarrow \infty} \int_{d}^{\infty} U(1, x) d x=0\right)\right)=1 .
$$

Therefore, we get

$$
\begin{aligned}
\nu_{T} & \left\{f: \lim _{d \rightarrow \infty} \int_{2 d}^{\infty} f(x) d x=0\right\} \\
& =\frac{1}{T} \int_{0}^{T} Q^{u_{0}}\left(\lim _{d \rightarrow \infty} \int_{2 d}^{\infty} V(t, x) d x=0\right) d t \\
& =\frac{1}{T} \int_{0}^{T} Q^{u_{0}}\left(\forall \varepsilon>0, \exists d_{0}, \int_{2 d}^{\infty} V(t, x) d x<\varepsilon \text { for } \forall d>d_{0}\right) d t \\
& \geq \frac{1}{T} \int_{1}^{T} Q^{u_{0}}\left(\forall \varepsilon>0, \exists d_{0}, \int_{d+R(t-1)}^{\infty} U(t, x) d x<\varepsilon,\right.
\end{aligned}
$$




$$
\begin{aligned}
& \left.|R(t)-R(t-1)| \leq d, \text { for } \forall d>d_{0}\right) d t \\
\geq & \frac{1}{T} \int_{1}^{T} Q^{u_{0}}\left(Q^{V(t-1)}\left(\lim _{d \rightarrow \infty} \int_{d}^{\infty} U(1, x) d x=0\right)\right) d t \\
& -\lim _{d \rightarrow \infty} Q^{\nu_{T}}(|R(1)| \geq d) .
\end{aligned}
$$

Lemma 3.5 implies that the second term on the right hand side of (3.88) tends to zero as $d \rightarrow \infty$. Together with (3.87) we get (3.81). Since $\nu_{T(n)}\{f:$ $\left.\int_{\mathbf{R}} f(x) e^{x} d x=1\right\}=1$, we have $\nu\left\{f: \int_{\mathbf{R}} f(x) e^{x} d x \leq 1\right\}=1$. Then

$$
\begin{aligned}
& \nu\left\{f: \int_{\mathbf{R}} f(x) e^{x} d x \geq 1\right\} \\
& \geq \nu\left\{f: \lim _{d \rightarrow \infty} \int_{\mathbf{R}} f(x) e^{d-|x-d|} d x \geq 1\right\} \\
& \quad \geq \limsup _{n \rightarrow \infty} \nu_{T(n)}\left\{f: \lim _{d \rightarrow \infty} \int_{\mathbf{R}} f(x) e^{d-|x-d|} d x=1\right\} \\
& =\limsup _{n \rightarrow \infty} \frac{1}{T(n)} \int_{0}^{T(n)} Q^{u_{0}}\left(\lim _{d \rightarrow \infty} \int_{\mathbf{R}} V(t, x) e^{d-|x-d|} d x=1\right) d t \\
& \quad=\limsup _{n \rightarrow \infty} \frac{1}{T(n)} \int_{0}^{T(n)} Q^{u_{0}}\left(\lim _{d \rightarrow \infty} \int_{d}^{\infty}\left(V(t, x) e^{2 d-x}-V(t, x) e^{x}\right) d x=0\right) d t \\
& =\limsup _{n \rightarrow \infty} \frac{1}{T(n)} \int_{0}^{T(n)} Q^{u_{0}}\left(\lim _{d \rightarrow \infty} \int_{d}^{\infty} V(t, x) d x=0\right) d t=1 .
\end{aligned}
$$

To prove (3.83), we have

$$
\begin{aligned}
Q^{\nu}(\exists t>0, U(t)=0) & \leq Q^{\nu}(R(t)<-d) \\
& \leq \liminf _{n \rightarrow \infty} Q^{\nu_{T(n)}}(R(t)<-d) \\
& \leq \frac{C^{\prime}}{d} \rightarrow 0 \text { as } d \rightarrow \infty
\end{aligned}
$$

Next we show that $u(t, \cdot+R(t))$ is a stationary process and $Q^{\nu}$ is the law of a traveling wave solution to (1.9). Let $F: C_{\text {tem }}^{+} \rightarrow \mathbf{R}$ be bounded and continuous. Suppose that $f_{n} \rightarrow f$. Denote $u_{n}$ the solution to (1.9) with initial condition $f_{n}$. Then it may be checked that the Kolmogorov tightness criterion holds and any limit point $u$ has law $Q^{f}$. This proves that $Q^{f_{n}} \rightarrow Q^{f}$. The continuity of $f \rightarrow Q^{f}$ gives that $Q^{\nu_{T(n)}} \rightarrow Q^{\nu}$. Therefore we get

$$
\begin{aligned}
Q^{\nu}(F(V(t))) & =\lim _{n \rightarrow \infty} Q^{\nu_{T(n)}}(F(V(t))) \\
& =\lim _{n \rightarrow \infty} \frac{1}{T(n)} \int_{0}^{T(n)} Q^{u_{0}}\left(Q^{V(s)}(F(V(t)))\right) d s \\
& =\lim _{n \rightarrow \infty} \frac{1}{T(n)} \int_{0}^{T(n)} Q^{u_{0}}(F(V(t+s))) d s \\
& =\nu(F) .
\end{aligned}
$$


We have shown that under $Q^{\nu}$, the one dimensional marginal of $\{V(t): t \geq 0\}$ has law $\nu$. It is straightforward to check that $\{V(t): t \geq 0\}$ is Markov. Hence $\{V(t): t \geq 0\}$ is stationary. This verifies that $Q^{\nu}$ is the law of a traveling wave solution to $(1.9)$.

\section{Asymptotic property of wavefront}

In this section, we consider the asymptotic property of the wavefront $R(t)$. We suppose that the limit

$$
k_{\infty}=\lim _{t \rightarrow \infty} \frac{1}{2 t} \int_{0}^{t} k^{2}(s) d s
$$

exists and $0 \leq k_{\infty} \leq c_{0}$. Denote $\gamma=\sqrt{D\left(2 c_{0}-2 k_{\infty}\right)}$. The following theorem shows the asymptotic property of $R(t)$.

Theorem 4.1. Suppose that the solution $u$ to (1.9) with initial condition $u_{0}(x)=$ $\chi_{(-\infty, 0]}(x)$ survives forever. Then

$$
\lim _{t \rightarrow \infty} \frac{R(t)}{t}=\gamma \text { a.s. }
$$

Proof. For any $h>0$, choose $0<\varepsilon<h^{2} / 2 D+\sqrt{2 D c_{0}-2 D k_{\infty}+D^{2}} h / D$. Then for a.e. $\omega \in \Omega$, there is $T_{1}$, such that

$$
\exp \left(-\frac{k^{2}}{2} t-\varepsilon t\right) \leq \mathcal{E}_{t}(k, \omega) \leq \exp \left(-\frac{k^{2}}{2} t+\varepsilon t\right)
$$

for $t \geq T_{1}$. So together with (2.6), we have

$$
\begin{aligned}
u(t, x) & \leq \exp \left(c_{0} t-\frac{1}{2} \int_{0}^{t} k^{2}(s) d s+\varepsilon t\right) \mathbb{E}\left[\chi_{(-\infty, 0]}\left(x+\sqrt{D} B_{t}\right)\right] \\
& =\exp \left(c_{0} t-\frac{1}{2} \int_{0}^{t} k^{2}(s) d s+\varepsilon t\right) \mathbb{P}\left(B_{t} \leq-\frac{x}{\sqrt{D}}\right) \\
& \leq \exp \left(c_{0} t-\frac{1}{2} \int_{0}^{t} k^{2}(s) d s+\varepsilon t-\frac{x^{2}}{2 D t}\right) \text { a.s. }
\end{aligned}
$$

for $t \geq T_{1}$. Let $x \geq(\beta+h) t$, where $\beta$ is a constant. Multiplying $e^{x}$ in (4.4) and integrating with respect to $x$ in $[(\beta+h) t, \infty)$, we have

$$
\begin{aligned}
\int_{(\beta+h) t}^{\infty} u(t, x) e^{x} d x \leq & \int_{(\beta+h) t}^{\infty} \exp \left(c_{0} t-\frac{1}{2} \int_{0}^{t} k^{2}(s) d s+\varepsilon t-\frac{x^{2}}{2 D t}+x\right) d x \\
= & \sqrt{2 D t} \exp \left(c_{0} t-\frac{1}{2} \int_{0}^{t} k^{2}(s) d s+\varepsilon t+\frac{D}{2} t\right) \\
& \times \int_{\frac{(\beta+h) t-D t}{\sqrt{2 D t}}}^{\infty} e^{-y^{2}} d y
\end{aligned}
$$




$$
\begin{aligned}
\leq & \frac{\sqrt{2 D t}}{2} \exp \left(\left(c_{0}+\varepsilon-\frac{\beta^{2}}{2 D}-\frac{\beta h}{D}-\frac{h^{2}}{2 D}-\beta-h\right) t\right) \\
& \times \exp \left(-\frac{1}{2} \int_{0}^{t} k^{2}(s) d s\right) \text { a.s. }
\end{aligned}
$$

for $t \geq T_{1}$. We let $\beta=\sqrt{2 D c_{0}-2 D k_{\infty}+D^{2}}-D$. Then we have

$$
\lim _{t \rightarrow \infty} \int_{(\beta+h) t}^{\infty} u(t, x) e^{x} d x=0 \text { a.s. }
$$

Similarly with the computation of (4.5), we have

$$
\begin{aligned}
\int_{(\gamma+h) t}^{(\beta-h) t} u(t, x) e^{x} d x \leq & \int_{(\gamma+h) t}^{(\beta-h) t} \exp \left(c_{0} t-\frac{1}{2} \int_{0}^{t} k^{2}(s) d s+\varepsilon t-\frac{x^{2}}{2 D t}+x\right) d x \\
= & \sqrt{2 D t} \exp \left(c_{0} t-\frac{1}{2} \int_{0}^{t} k^{2}(s) d s+\varepsilon t+\frac{D}{2} t\right) \\
& \times \int_{\frac{(\gamma+h) t-D t}{\sqrt{2 D t}}}^{\frac{(\beta-h) t-D t}{\sqrt{2 D t}}} e^{-y^{2}} d y \\
\leq & \frac{\sqrt{2 D t}}{2} \exp \left(c_{0} t-\frac{1}{2} \int_{0}^{t} k^{2}(s) d s+\varepsilon t\right) \\
& \times \exp \left(-\frac{\gamma^{2}}{2 D} t-\frac{\gamma h}{D} t-\frac{h^{2}}{2 D} t+\gamma t+h t\right) \\
& -\frac{\sqrt{2 D t}}{2} \exp \left(c_{0} t-\frac{1}{2} \int_{0}^{t} k^{2}(s) d s+\varepsilon t\right) \\
& \times \exp \left(-\frac{\beta^{2}}{2 D} t+\frac{\beta h}{D} t-\frac{h^{2}}{2 D} t+\beta t-h t\right) \text { a.s. }
\end{aligned}
$$

for $t \geq T_{1}$. From the definition of $\gamma$ and $\beta$, (4.7) may reduce to

$$
\begin{aligned}
\int_{(\gamma+h) t}^{(\beta-h) t} u(t, x) e^{x} d x \leq & \frac{\sqrt{2 D t}}{2} \exp \left(k_{\infty} t-\frac{1}{2} \int_{0}^{t} k^{2}(s) d s+\varepsilon t\right) \\
& \times \exp \left(\gamma t-\frac{\gamma h t}{D}-\frac{h^{2} t}{2 D}+h t\right) \\
& -\frac{\sqrt{2 D t}}{2} \exp \left(k_{\infty} t-\frac{1}{2} \int_{0}^{t} k^{2}(s) d s+\varepsilon t\right) \\
& \times \exp \left(\frac{\beta h t}{D}-\frac{h^{2} t}{2 D}-h t\right) \text { a.s. }
\end{aligned}
$$

for $t \geq T_{1}$. Analogously to the derivation of (4.8), we get

$$
\begin{aligned}
\int_{(\gamma-h) t}^{(\gamma+h) t} u(t, x) e^{x} d x \leq & \frac{\sqrt{2 D t}}{2} \exp \left(k_{\infty} t-\frac{1}{2} \int_{0}^{t} k^{2}(s) d s+\varepsilon t\right) \\
& \times \exp \left(\gamma t+\frac{\gamma h t}{D}-\frac{h^{2} t}{2 D}-h t\right)
\end{aligned}
$$




$$
\begin{aligned}
& -\frac{\sqrt{2 D t}}{2} \exp \left(k_{\infty} t-\frac{1}{2} \int_{0}^{t} k^{2}(s) d s+\varepsilon t\right) \\
& \times \exp \left(\gamma t-\frac{\gamma h t}{D}-\frac{h^{2} t}{2 D}+h t\right) \text { a.s. }
\end{aligned}
$$

and

$$
\begin{aligned}
\int_{(\beta-h) t}^{(\beta+h) t} u(t, x) e^{x} d x \leq & \frac{\sqrt{2 D t}}{2} \exp \left(k_{\infty} t-\frac{1}{2} \int_{0}^{t} k^{2}(s) d s+\varepsilon t\right) \\
& \times \exp \left(\frac{\beta h t}{D}-\frac{h^{2} t}{2 D}-h t\right) \\
& -\frac{\sqrt{2 D t}}{2} \exp \left(k_{\infty} t-\frac{1}{2} \int_{0}^{t} k^{2}(s) d s+\varepsilon t\right) \\
& \times \exp \left(-\frac{\beta h t}{D}-\frac{h^{2} t}{2 D}+h t\right) \text { a.s. }
\end{aligned}
$$

for $t \geq T_{1}$. By (1.11) we know that there exists a $T_{2}>0$ such that for $t \geq T_{2}$, $x<(\gamma-h) t$,

$$
\exp \left(-c_{3} \sqrt{2 t \ln \ln t}\right) \leq u(t, x) \leq \exp \left(c_{2} \sqrt{2 t \ln \ln t}\right) \text { a.s. }
$$

which implies that

$$
\int_{-\infty}^{(\gamma-h) t} u(t, x) e^{x} d x \leq \exp \left(c_{2} \sqrt{2 t \ln \ln t}+\gamma t-h t\right) \text { a.s. }
$$

for $t \geq T_{2}$. From (4.6) there is a $T_{3}>0$ such that $\int_{(\beta+h) t}^{\infty} u(t, x) e^{x} d x \leq 1$ for $t \geq T_{3}$. Then associating with (4.8), (4.9), (4.10) and (4.12), we get

$$
\int_{\mathbf{R}} u(t, x) e^{x} d x \leq \exp \left(c_{2} \sqrt{2 t \ln \ln t}+\gamma t-h t\right)(2+H(t)+G(t)) \text { a.s., }
$$

where

$$
\begin{aligned}
H(t)= & \frac{\sqrt{2 D t}}{2} \exp \left(k_{\infty}-\frac{1}{2} \int_{0}^{t} k^{2}(s) d s+\varepsilon t\right) \\
& \times \exp \left(\frac{\beta h t}{D}-\frac{h^{2} t}{2 D}-c_{2} \sqrt{2 t \ln \ln t}-\gamma t\right)
\end{aligned}
$$

and

$$
\begin{aligned}
G(t)= & \frac{\sqrt{2 D t}}{2} \exp \left(k_{\infty}-\frac{1}{2} \int_{0}^{t} k^{2}(s) d s+\varepsilon t\right) \\
& \times \exp \left(-\frac{\gamma h t}{D}-\frac{h^{2} t}{2 D}+2 h t-c_{2} \sqrt{2 t \ln \ln t}\right) .
\end{aligned}
$$


By the arbitrariness of $h$ and $\varepsilon$, there is a $T_{4}>0$ such that $H(t) \leq 1$ a.s. for $t \geq T_{4}$. On the other hand, we have

$$
\begin{aligned}
\frac{1}{t} \ln G(t)= & \frac{1}{2 t} \ln 2 D t-\frac{1}{t} \ln 2+\frac{1}{t}\left(k_{\infty}-\frac{1}{2} \int_{0}^{t} k^{2}(s) d s\right) \\
& +\varepsilon-\frac{\gamma h}{D}-\frac{h^{2}}{2 D}+2 h-\frac{1}{t} c_{2} \sqrt{2 t \ln \ln t} \text { a.s. }
\end{aligned}
$$

By the arbitrariness of $h$ and $\varepsilon,(4.16)$ implies

$$
\lim _{t \rightarrow \infty} \frac{1}{t} \ln G(t)=0 \text { a.s. }
$$

So (4.13) gives that

$$
\frac{1}{t} R(t) \leq \frac{1}{t} c_{2} \sqrt{2 t \ln \ln t}+\gamma-h+\frac{1}{t} \ln 8+\frac{1}{t} \ln G(t) \text { a.s. }
$$

which implies that

$$
\limsup _{t \rightarrow \infty} \frac{1}{t} R(t) \leq \gamma \text { a.s. }
$$

By (4.11), a straightforward estimation shows that

$$
\frac{1}{t} R(t) \geq-\frac{1}{t} c_{3} \sqrt{2 t \ln \ln t}+\gamma-h \text { a.s. }
$$

Since the arbitrariness of $h,(4.20)$ implies that

$$
\liminf _{t \rightarrow \infty} \frac{1}{t} R(t) \geq \gamma \text { a.s. }
$$

Associating with (4.19) and (4.21) gives

$$
\lim _{t \rightarrow \infty} \frac{1}{t} R(t)=\gamma \text { a.s. }
$$

which completes the proof of the theorem.

Theorem 4.1 implies that the wavefront $R(t)$ coincides with the wavefront obtained in $[12,34]$ after sufficiently large time. Thus the theorem suggests that the choice of the wavefront in Sect. 2 is suitable.

\section{References}

[1] Arnold, L.: Random Dynamical System, Springer, New York (1998)

[2] Ablowitz, M.J., Zeppetella, A.: Explicit solutions of Fisher's equation for a special wave speed. Bull. Math. Biol. 41, 835-840 (1979)

[3] Ai, S.: Traveling wave fronts for generalized Fisher equations with spatiotemporal delays. J. Differ. Equ. 232, 104-133 (2007)

[4] Bramson, M.: Convergence of solutions of the Kolmogorov equation to travelling waves. Mem. Amer. Math. Soc. 44, 285 (1983) 
[5] Bressloff, P.C., Webber, M.A.: Front propagation in stochastic neural fields. SIAM J. Appl. Dynam. Syst. 11, 708-740 (2012)

[6] Bates, P.W., Lu, K., Wang, B.: Random attractors for stochastic reactionCdiffusion equations on unbounded domains. J. Differ. Equ. 246, 845-869 (2009)

[7] Conlon, J.G., Doering, C.R.: On travelling waves for the stochastic FisherKolmogorov-Petrovsky-Piscunov equation. J. Stat. Phy. 144, 137-177 (2009)

[8] Cerrai, S., Freidlin, M.: Averaging principle for a class of stochastic reactiondiffusion equations. Probab. Theory Relat. Fields 120, 421-477 (2005)

[9] Chen, B., Xie, Y.: Exact solutions for generalized stochastic Wick-type KdVmKdV equations. Chaos Solitons Fract. 23, 281-287 (2005)

[10] Davies, L.M., Truman, A., Zhao, H.: Stochastic generalized KPP equations. Proc. R. Soc. Edinb. A 126, 957-983 (1996)

[11] Doering, C.R., Mueller, C., Smereka, P.: Interacting particles, the stochastic Fisher-Kolmogorov-Petrovsky-Piscunov equation and duality. Physica A 325, 243-259 (2003)

[12] Elworthy, K.D., Zhao, H.: The propagation of travelling waves for stochastic generalized KPP equations. Math. Comput. Modelling 20, 131-166 (1994)

[13] Fisher, R.A.: The wave of advance of advantageous gene. Ann. Eugen. 7, 355369 (1937)

[14] Feldman, M.W., Roughgarden, J.: A population's stationary distribution and chance of extinction in stochastic environment with remarks on the theory of species packing. Theor. Popul. Biol. 7, 197-207 (1975)

[15] Gomez, A., Trofimchuk, S.: Monotone traveling wavefronts of the KPP-Fisher delayed equation. J. Differ. Equ. 250, 1767-1787 (2011)

[16] Herman, R.L.: The Stochastic, damped KdV equation. J. Phys. A 23, 1063$1084(1990)$

[17] Holden, H.; Øksendala, B.; Ubøe, J.; Zhang, T: Stochastic Partial Differential Equations, Birkhäuser, Boston (1996)

[18] Han, X., Shen, W., Zhou, S.: Random attractors for stochastic lattice dynamical systems in weighted spaces. J. Differ. Equ. 250, 1235-1266 (2011)

[19] Kotelenez, P.: Comparison methods for a class of function valued stochastic partial differential equations. Probab. Theory Relat. Fields 93, 1-19 (1992)

[20] Kolmogorov, A., Petrovsky, I., Piscunov, N.: Study of adiffusion equation that is related to the growth of a quality of matter, and its application to a biological problem. Byul. Mosk. Gos. Univ. Ser. A Mat. Mekh. 1, 1-26 (1937)

[21] Keiding, N.: Extinction and exponential growth in random environments. Theor. Popul. Biol. 8, 49-63 (1975) 
[22] Konno, N., Shiga, T.: Stochastic partial differential equations for some measurevalued diffusions. Probab. Theory Relat. Fields 79, 201-225 (1988)

[23] Kiester, A.R.; Barakat, R.: Exact solutions to certain stochastic differential equation models of population growth. Theor. Popul. Biol. 6, 199-216 (1974)

[24] Lau, K.S.: On the nonlinear diffusion equation of Kolmogorov, Petrovsky and Piscounov. J. Differ. Equ. 59, 44-70 (1985)

[25] Lungu, E.M., Øksendal, B.: Optimal harvesting from a population in stochastic crowded environment. Math. Biosci. 145, 47-75 (1997)

[26] Li, D., He, D., Xu, D.: Mean square exponential stability of impulsive stochastic reaction-diffusion CohenCGrossberg neural networks with delays. Math. Comput. Simul. 82, 1531-1543 (2012)

[27] Long, S., Xu, D.: Stability analysis of stochastic fuzzy cellular neural networks with time-varying delays. Neurocomputing 74, 2385-2391 (2011)

[28] Lu, K., Schmalfu $\beta$, B.: Invariant manifolds for stochastic wave equations. J. Differ. Equ. 236, 460-492 (2007)

[29] Mueller, C., Sowers, R.B.: Random travelling waves for the KPP equation with noise. J. Funct. Anal. 128, 439-498 (1995)

[30] Mueller, C., Mytnik, L., Quastel, J.: Small noise asymptotics of traveling waves. Markov Process. Relat. Fields 14, 333-342 (2008)

[31] Mueller, C., Perkins, E.A.: The compact support property for solutions to the heat equation with noise. Probab. Theory Relat. Fields 93, 325-358 (1992)

[32] May, R.M.: Stability in randomly fluctuating versus deterministic environments. Am. Nat. 10, 621-650 (1973)

[33] May, R.M.: Stability and Complexity in Model Ecosystems. Princeton University Press, New Jersey (1974)

[34] Øksendala, B., Våge, G., Zhao, H.: Asymptotic properties of the solutions to stochastic KPP equations. Proc. R. Soc. Edinb. A 130, 1363-1381 (2000)

[35] Øksendala, B., Våge, G., Zhao, H.: Two properties of stochastic KPP equations: ergodicity and pathwise property. Nonlinearity 14, 639-662 (2001)

[36] Pan, S.: Asymptotic behavior of traveling fronts of the delayed Fisher equation. Nonlinear Anal. Real World Appl. 10, 1173-1182 (2009)

[37] Raugel, G., Kirchgässner, K.: Stability of fronts for a KPP-system. II. The critical case. J. Differ. Equ. 146, 399-456 (1998)

[38] Shen, W.: Traveling waves in diffusive random media. J. Dynam. Differ. Equ. 16, 1011-1059 (2004)

[39] Tribe, R.: A travelling wave solution to the Kolmogorov equation with noise. Stoch. Stoch. Rep. 56, 317-340 (1995) 
[40] Tribe, R., Woodward, N.: Stochastic order methods applied to stochastic travelling waves. Electron. J. Probab. 16, 436-469 (2011)

[41] Tuckwell, H.C.: A study of some diffusion models of population growth. Theor. Popul. Biol. 5, 345-357 (1974)

[42] Wadati, M.: Stochastic Korteweg-de Vries equation. J. Phys. Soc. Jpn. 52, 2642$2648(1983)$

[43] Wei, C., Wang, J.: Travelling wave solutions to the generalized stochastic KdV equation. Chaos. Solitons Frac. 37, 733-740 (2008)

[44] Xie, Y.: Exact solutions for stochastic KdV equations. Phy. Lett. A 310, 161$167(2003)$

[45] Yanagida, E.: Irregular behavior of solutios for Fisher's equation. J. Dynam. Differ. Equ. 19, 895-914 (2007)

[46] Zou, X.: Delay induced traveling wave fronts in reaction diffusion equations of KPP-Fisher type. J. Comput. Appl. Math. 146, 309-321 (2002)

[47] Zhou, S., Wei, L.: A random attractor for a stochastic second order lattice system with random coupled coefficients. J. Math. Anal. Appl. 395, 42-55 (2012)

Zhehao Huang and Zhengrong Liu

Department of Mathematics

South China University of Technology

Guangzhou, 510640

Guangdong

People's Republic of China

e-mail: zhehao.h@mail.scut.edu.cn

Zhengrong Liu

e-mail: liuzhr@scut.edu.cn

Received: 12 January 2014.

Accepted: 3 June 2014. 